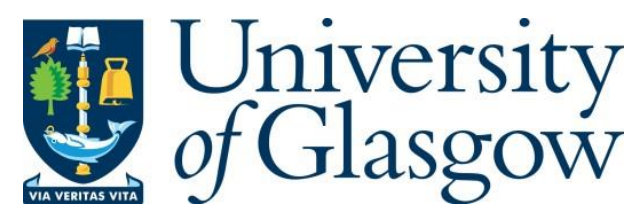

Mohjazi, L., Muhaidat, S., Dianati, M. and Al-Qutayri, M. (2018) Performance analysis of SWIPT relay networks with noncoherent modulation.IEEE Transactions on Green Communications and Networking, 2(4), pp. 1072-1086.

There may be differences between this version and the published version. You are advised to consult the publisher's version if you wish to cite from it.

$\underline{\text { http://eprints.gla.ac.uk/223103/ }}$

Deposited on: 10 September 2020

Enlighten - Research publications by members of the University of Glasgow http://eprints.gla.ac.uk 


\title{
Performance Analysis of SWIPT Relay Networks with Noncoherent Modulation
}

\author{
Lina Mohjazi, Student Member, IEEE, Sami Muhaidat, Senior Member, IEEE, Mehrdad Dianati, Senior \\ Member, IEEE, and Mahmoud Al-Qutayri, Senior Member, IEEE
}

\begin{abstract}
In this paper, we investigate the performance of noncoherent modulation in simultaneous wireless information and power transfer (SWIPT) relay networks. Noncoherent modulation schemes eliminate the need for instantaneous channel state information (CSI) estimation, and therefore, minimise the overall energy consumption of the network. In particular, we adopt a moments-based approach to develop a comprehensive novel analytical framework for the analysis of the outage probability, achievable throughput, and average symbol error rate (ASER) of a dual-hop SWIPT relay system considering the time switching (TS) and power splitting (PS) receiver architectures. In addition, through the derivation of new asymptotic analytical results for the outage probability and ASER, we analytically demonstrate that the diversity order of the considered system is non-integer less than 1 in the high SNR regime. Our results show that there is a unique value for the PS ratio that minimises the outage probability of the system, while this is not the case for the TS protocol. We also demonstrate that, in terms of system throughput, the TS relaying scheme is superior to the PS relaying scheme at lower SNR values. An extensive Monte Carlo simulation study is presented to corroborate the proposed analytical model.
\end{abstract}

Index Terms-Noncoherent modulation, relay networks, simultaneous wireless information and power transfer.

\section{INTRODUCTION}

$\mathbf{S}$ IMULTANEOUS wireless information and power transfer (SWIPT) is an emerging technology which was proposed to prolong the lifetime of energy-constrained wireless networks and to provide an alternative solution to limited lifetime battery-operated devices. In this context, designing efficient transmission mechanisms and protocols for SWIPT has been the recent focus of intensive research, due to its promising applications in the next generation wireless networks [2]-[4]. With SWIPT, a wireless terminal is powered up by a received radio frequency (RF) signal and, simultaneously, information processing is carried out using the same signal [5].

SWIPT-based relaying was proposed as a promising technique to provide throughput improvement, communication reliability enhancement, and coverage range extension [6]. From this perspective, the theoretical and implementation

This work was accepted in part at the IEEE VTC Fall, Toronto, Canada, 2017 [1].

L. Mohjazi and S. Muhaidat are with the Institute for Communication Systems, Department of Electronic Engineering, University of Surrey, Surrey, U.K. (e-mail: 1.mohjazi@surrey.ac.uk, muhaidat@ieee.org).

M. Dianati is with the Warwick Manufacturing Group, University of Warwick, Coventry, U.K. (e-mail: m.dianati@warwick.ac.uk).

S. Muhaidat and M. Al-Qutayri are with the Department of Electrical and Computer Engineering, Khalifa University, Abu Dhabi, UAE (e-mail: muhaidat@ieee.org, mqutayri@kustar.ac.ae). aspects of SWIPT relay networks have been areas of active research interest (see [7]-[10] and the references therein). A SWIPT-enabled relay terminal harvests energy from the signal transmitted from the source node to forward the information to a destination node. Owing to practical hardware limitations, a relay node is unable to perform the two functions of energy harvesting (EH) and information processing simultaneously, an approach called the ideal receiver [5]. To address this problem, two practically realisable SWIPT relaying protocols were proposed in [11], namely, time switching (TS) and power splitting (PS) relaying protocols. In the former, the relay switches over time between EH and information processing, whereas, in the latter, the relay uses portion of the received power for $\mathrm{EH}$ and the remaining for information processing. One of the key challenges in SWIPT relay systems is that RF-powered relay terminals have a stringent power constraint which limits the use of high power consumption algorithms. Therefore, not only channel coding, routing protocols, and receiver operation policies need to be redesigned for RFpowered relay communications, but also, employing energy efficient modulation techniques is crucial for the design of SWIPT relay systems [12].

Although there has been a growing literature on SWIPT, particularly, in the context of relay networks, (see [6]-[10] and the references therein), all research studies were built upon the assumption of perfect channel state information (CSI) knowledge to allow for coherent information delivery. Smart world is envisioned as an era in which billions of objects, such as sensors, are connected to a common interface with the ability to automatically and intelligently serve people in a collaborative-aware manner [13]. The basis of this vision is to connect a massive number of low-power small-size devices that incorporate sensing, computation and communication capabilities. In this regard, several fundamental considerations need to be taken into account, in order to successfully support various requirements, such as low deployment cost, long battery life, and low device cost. Noncoherent modulation are simple schemes that eliminate the need of CSI between the connecting objects. Due to the absence of the channel estimation procedure, the hardware complexity of the receiver is reduced and consequently, the power efficiency is increased. This renders noncoherent modulation to be an attractive practical solution and is currently adopted in many standards, such as in IEEE 802.11 [14].

Recently, noncoherent modulation techniques for SWIPT relay systems have been proposed in [15]-[20] to circumvent channel estimation. In particular, in [15], [16], maximum- 
likelihood detectors were obtained based on TS and PS SWIPT receiver architectures, for amplify-and-forward (AF) and decode-and-forward (DF) relaying, respectively. The performance of the proposed receivers in terms of their average symbol error rate (ASER) was studied via Monte-Carlo simulations only. Furthermore, noncoherent modulation for two-way relay networks was investigated in [17]. In [18] and [19], the authors investigated the error rate performance of differential modulation in SWIPT-based AF relaying over Nakagami- $m$ and Rayleigh fading channels, respectively. The derived expressions for the average bit error rate (ABER) are applicable only for the case of binary differential phaseshift keying (BDPSK). In [20], we derived bounds on the ABER of SWIPT AF relay networks, employing the PS relaying protocol, for BDPSK. In our analysis, we ignored the dependency of the relay-to-destination hop on the sourceto-relay hop resulting from the PS at the relay. Our results demonstrated that the bound is tight in the low to medium signal-to-noise (SNR) regime and that there is no loss in the ABER performance in this region.

Although analysing the performance and understanding the limitations of noncoherent SWIPT relay networks in various operational conditions is essential for their design, implementation, testing and deployment stages, to the best of our knowledge, none of the previous works provided a comprehensive analytical treatment to efficiently evaluate the performance of such systems.

It is known that DF relaying incurs extra energy consumption resulting from information decoding at the relay and that AF relaying enjoys a simple operation involving lowcomplexity receiver circuitry [21] making it more attractive in RF-powered relay nodes. Considering this fact and motivated by the above considerations, the aim of this paper is, therefore, to fill this research gap by developing a comprehensive mathematical framework to analytically study the performance of arbitrary $M$-ary noncoherent frequency-shift keying ( $M$ FSK) and $M$-ary differential phase-shift keying ( $M$-DPSK) in dual-hop AF relaying protocols with TS and PS SWIPT schemes. We also investigate the system performance with an ideal relay receiver as a benchmark relaying scheme, that is based on the ideal receiver in [5], which processes the information and extracts power from the same received signal. Different from the work presented in [20], in this work, we consider the assumption of a general SWIPT relay system, where the SNRs of the two hops are correlated. Also, unlike [18]-[20], where the analysis therein is limited to the ABER, in this work we adopt the moments-based approach to evaluate various performance metrics that quantify the operation of SWIPT relay networks. This includes, but is not limited to, the outage probability, the achievable throughput, the ASER, and the diversity order.

Specifically, the main contributions and results of this paper are summarised as follows:

- We derive a computationally effective exact closed-form expression for the moments of the receive SNR when the channels of the two hops are subject to Rayleigh fading. To the best of our knowledge, this result is novel in literature.
- We employ the Padé approximation (PA) technique to obtain an accurate approximate closed-form rational expression of the corresponding moment generating function (MGF) of the system based on the knowledge of its moments.

- The derived moments and MGF expressions are utilised to evaluate fundamental performance metrics such as, the average SNR (ASNR), the amount-of-fading (AoF), the outage probability, the achievable throughput, and the ASER for the cases of arbitrary $M$-FSK and $M$-DPSK.

- We derive new asymptotic expressions for the outage probability and the ASER, respectively.

- Through the derived asymptotic outage probability expression, we demonstrate that the achievable diversity order of the considered system model is non-integer less than 1 and further demonstrate that the second hop is indeed the performance bottleneck for the relaying path.

- The accuracy of all developed analytical models are verified via computer-based Monte Carlo simulations.

The remainder of the paper is organised as follows. In Section II, the system model and assumptions are specified. In Section III, analytical closed-form and approximate expressions for the moments and the MGF of the receive SNR are derived, respectively. The evaluations of ASNR, AoF, outage probability, achievable throughput, and ASER are presented in Section IV. We provide a thorough asymptotic analysis of the considered system model in Section V. Simulation results are illustrated in Section VI to corroborate the developed analytical models and to investigate the effect of various system parameters on the performance. Some concluding remarks are given in Section VII.

Notation: Bold lower case letters denote vectors and lower case letters denote scalers. A circularly symmetric complex Gaussian random variable $z$ with mean $\mu$ and variance $\sigma^{2}$ is represented as $\mathcal{C N}\left(\mu, \sigma^{2}\right)$. Also, $\mathbf{I}_{N}$ and $\mathbf{i}_{n}$ denote the identity matrix of size $N$ and a column vector with 1 at its $n$-th entry and 0 elsewhere, respectively. Moreover, $(.)^{T}, \mathbb{E}[z],|z|, \Re\{$.$\} ,$ and $\ln ($.$) stand for the transpose, expectation of the random$ variable $z$, magnitude of a complex variable $z$, real part, and natural logarithm, respectively.

\section{SySTEM MODEL}

Consider an AF wireless relay system where a source node, $S$, communicates with a destination node, $D$, via an intermediate relay node, $R$. Let $h_{s r}$ and $h_{r d}$ denote the channel coefficients of the first and the second hops, respectively. The source and the destination nodes are assumed to be energy unconstrained nodes powered by either a battery or a power grid. Furthermore, the source transmits its message using a constant transmit power, $P_{s}$. On the other hand, the relay node has no dedicated power supply and harvests energy from the received signal of $S$, which is then used as a transmit power over the second hop. Our study considers two wireless EH protocols, namely, PS and TS, and assumes that all nodes are equipped with a single antenna. We further consider that a direct link does not exist between $S$ and $D$. This is motivated by the fact that the direct source to destination link is assumed 
to be strongly attenuated, due to deep fading, or shadowing effects, due to surrounding physical obstacles, or both and communication can be established only via a relay node. Such an assumption is widely adopted in research studies related to SWIPT relay communication [6], [7], [17]. Also, this assumption corresponds to coverage extension scenarios where the distance between the source and destination is long and relays are used in order to maintain connectivity [13]. This scenario is envisioned in furture Internet-of-Things (IoTs) deployments, where RF-powered relays are employed to provide coverage expansion To avoid interference, data transmission from $S$ and $R$ are performed over orthogonal channels in either frequency or time. For ease of exposition, we consider a time division multiple access (TDMA) based transmission scheme, which was initially proposed for conventional relay systems with coherent detection [22] and was later applied to noncoherehnt/differiential systems [23]. All nodes are assumed to operate in half-duplex mode. That is, the signal transmission from $S$ to $D$ is completed in two phases. In Phase-1, the source to relay information transmission takes place. During Phase-2, $S$ remains silent, whereas $R$ uses the energy harvested during Phase-1 to amplify the received signal and forward it to $D$.

In our setup, $h_{i j}, i j \in\{s r, r d\}$, denotes the small-scale fading coefficient of the channel between transmitter $i$ and receiver $j$, and is modelled as independent complex Gaussian random variable, $h_{i j} \sim \mathcal{C N}\left(0, \sigma_{i j}^{2}\right)$. Moreover, we assume that the instantaneous CSIs of $h_{i j}$ are unknown to any terminal. Furthermore, we consider that all links are subject to large scale fading in which the received power is inversely proportional to $d_{i j}^{\alpha}$, where $d_{i j}$ is the distance between transmitter $i$ and receiver $j$, and $\alpha>2$ denotes the path-loss exponent. We also assume that information transfer is performed using noncoherent signalings such as $M$-FSK or $M$-DPSK, where $M$ is the constellation size.

\section{A. Power Splitting-Based Relaying}

The communication block diagram of the employed PS relaying protocol for SWIPT is depicted in Fig. 1. The total transmission time, $T$, is divided equally into two consecutive phases, each of duration $T / 2$ (sec) [6], [15]. Specifically, during Phase-1, the source broadcasts its signal $x_{m}$, where $\mathbb{E}\left[\left|x_{m}(n)\right|^{2}\right]=1$, with power $P_{s}$ (watts) according to one of the two following noncoherent modulation schemes.

1) PS with M-DPSK: In this case, the information symbols, $v(n)=e^{j 2 \pi m / M}$ for $m=0,1, \ldots, M-1$, are encoded based on the phase difference of two consecutive signals. Consequently, the source transmitted signal, $x_{m}, m=0,1, \ldots, M-1$, can be expressed as

$$
x_{m}(n)=x_{m}(n-1) v(n), n=1,2, \ldots N,
$$

where the initial reference modulated symbol is $x_{m}(0)=1$, and $N$ is the frame length [24]. More specifically, in the first time slot, the signal received at $R$ can be expressed as

$$
y_{r}(n)=\frac{\sqrt{P_{s}}}{\sqrt{d_{s r}^{\alpha}}} h_{s r} x_{m}(n)+w_{r a}(n)
$$

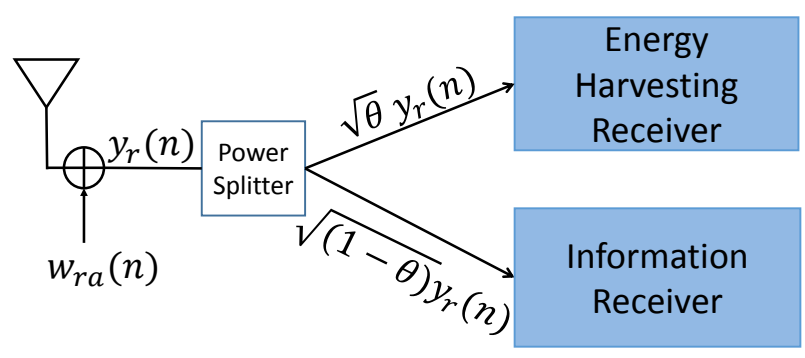

Fig. 1: Block diagram of the relay receiver architecture with the PS relaying protocol.

Here, $w_{r a}(n) \sim \mathcal{C N}\left(0, N_{0_{r a}}\right)$ represents the additive white Gaussian noise (AWGN) associated with the $n$th symbol, accounting for the receive antenna noise at $R$. At the end of Phase-1, $R$ splits the received signal into two streams, as shown in Fig. 1, one is forwarded to the energy harvester and the other to the information receiver (IR) for information processing. Thus, the signal received at IR of $R$ can be given by

$y_{s r}(n)=\sqrt{\kappa} y_{r}(n)=\frac{\sqrt{\kappa P_{s}}}{\sqrt{d_{s r}^{\alpha}}} h_{s r} x_{m}(n)+\sqrt{\kappa} w_{r a}(n)+w_{r c}(n)$,

where $\kappa=(1-\theta)$ and $\theta$ stands for the PS ratio at $R$. In this paper, it is considered that $0<\theta<1$, corresponding to a general SWIPT system featuring both wireless information transfer and wireless EH. Furthermore, $w_{r c}(n) \sim \mathcal{C N}\left(0, N_{0_{r c}}\right)$ is the AWGN at $R$ due to the RF to baseband signal conversion.

The remaining portion of the received signal at $R$ is forwarded to the energy harvester, i.e. $y_{\mathrm{EH}}(n)=\sqrt{\theta} y_{r}(n)$, hence, the overall energy harvested during Phase-1 can be expressed as

$$
E_{r}=\frac{\eta \theta P_{s}\left|h_{s r}\right|^{2}}{d_{s r}^{\alpha}}(T / 2),
$$

with $0<\eta<1$ denoting the energy conversion efficiency factor. Since $R$ communicates with $D$ for $T / 2$ sec., the power available at $R$ at the end of Phase-1 is $P_{r}=E_{r} /(T / 2)$.

During Phase-2, to simplify the analysis, $R$ is assumed to use the whole portion of the harvested energy to amplify the received signal, given in (3), and transmit it to $D$. At the end of Phase-2, the destination implements differential decoding to recover the data symbols from the received signal, which can be expressed as

$$
y_{r d}(n)=\frac{1}{\sqrt{d_{r d}^{\alpha}}} h_{r d} s_{r}(n)+w_{r d}(n),
$$

where $w_{r d}(n) \sim \mathcal{C N}\left(0, N_{0_{r d}}\right)$ is the overall AWGN at $D$. In addition, $w_{r a}(n), w_{r c}(n)$, and $w_{r d}(n)$ are statistically independent. In (5), $s_{r}(n)$ represents the signal transmitted from the relay after proper amplification. To ensure that the average transmit power of $R$ is $P_{r}$, the relay normalises the signal to be transmitted by the variance of (3) and $s_{r}(n)$ can 
be correspondingly, given as

$$
s_{r}(n)=\sqrt{\frac{P_{r}}{\frac{1}{d_{s r}^{\alpha}} \kappa P_{s} \sigma_{s r}^{2}+\kappa N_{0_{r a}}+N_{0_{r c}}}} y_{s r}(n) .
$$

We note that, the power scaling factor in (6), i.e., $\left(\frac{1}{d_{s r}^{\alpha}} \kappa P_{s} \sigma_{s r}^{2}+\right.$ $\left.\kappa N_{0_{r a}}+N_{0_{r c}}\right)^{1 / 2}$ ensures that the average transmission power for data relaying in Phase-2 is fixed to $P_{r}$ and relies solely on the variance of the channel $h_{s r}$ without requiring the knowledge of its CSI at the relay terminal [6], [15]. It should be further noted that the $\mathrm{EH}$ process at $R$ is independent of the power scaling process and it is assumed that $\mathrm{EH}$ is performed instantaneously. We would like to point out that the harvested instantaneous energy depends on the instantaneous power gain, namely, $\left|h_{s r}\right|^{2}$, but it does not require the knowledge of CSI. The harvested instantaneous energy is simply used as a transmit power in the second phase of transmission. Note that the assumption of instantaneous EH was adopted in [6] ${ }^{1}$. Finally, substituting (3) and (6) in (5) yields

$$
\begin{aligned}
y_{r d}(n) & =\underbrace{\frac{\sqrt{\kappa \eta \theta\left|h_{s r}\right|^{2}} P_{s} h_{s r} h_{r d} x_{m}(n)}{\sqrt{d_{s r}^{\alpha} d_{r d}^{\alpha}} \sqrt{\kappa P_{s} \sigma_{s r}^{2}+d_{s r}^{\alpha} N_{0_{s r}}}}}_{\text {Signal Part }} \\
& +\underbrace{\frac{\sqrt{\eta \theta P_{s}\left|h_{s r}\right|^{2}} h_{r d} w_{s r}(n)}{\sqrt{d_{r d}^{\alpha}} \sqrt{\kappa P_{s} \sigma_{s r}^{2}+d_{s r}^{\alpha} N_{0_{s r}}}}+w_{r d}(n)}_{\text {Noise Part }},
\end{aligned}
$$

where $w_{s r}(n)=\sqrt{\kappa} w_{r a}(n)+w_{r c}(n)$ is the overall noise at $R$ with variance $N_{0_{s r}}=\kappa N_{0_{r a}}+N_{0_{r c}}$. Based on (7), the instantaneous received SNR at $D, \gamma_{e q}$, can be given by

$$
\gamma_{e q}=\frac{a b \rho_{s r} \rho_{r d}\left|h_{s r}\right|^{4}\left|h_{r d}\right|^{2}}{b \rho_{r d}\left|h_{s r}\right|^{2}\left|h_{r d}\right|^{2}+a \rho_{s r} \sigma_{s r}^{2}+1},
$$

where $a=\frac{\kappa}{d_{s r}^{\alpha}}, b=\frac{\eta \theta}{d_{s r}^{\alpha} d_{r d}^{\alpha}}, \rho_{s r}=\frac{P_{s}}{N_{0_{s r}}}$ and $\rho_{r d}=\frac{P_{s}}{N_{0_{r d}}}$.

2) PS with noncoherent $M$-FSK: For noncoherent ${ }^{2} M$-ary FSK modulation, the source transmits the message, $x_{m}(n)$, over the $m$ th orthogonal carrier chosen from an $M$ set of carriers where $m=0,1, \ldots, M-1$. Since at the destination, the source message is detected based on $M$ received symbols, it is convenient to represent $x_{m}(n)$ as a column vector $\mathbf{i}_{m+1}$ with 1 at its $m$ th entry and 0 elsewhere. Moreover, the destination employs noncoherent reception using a bank of $M$ noncoherent correlators to make a decision as to which of the $M$ symbols was transmitted [24]. The baseband equivalent signal at any receiving terminal, $j$ is denoted by an $M \times 1$ vector $\mathbf{y}_{i j} \triangleq\left[y_{i j}(1), \ldots, y_{i j}(M)\right]^{T}$. As a result, the signal model for PS relaying employing noncoherent $M$-FSK can be represented as

$$
\begin{gathered}
\mathbf{y}_{s r}(n)=\frac{\sqrt{\kappa P_{s}}}{\sqrt{d_{s r}^{\alpha}}} h_{s r} \mathbf{i}_{m+1}(n)+\sqrt{\kappa} \mathbf{w}_{r a}(n)+\mathbf{w}_{r c}(n), \\
\mathbf{y}_{r d}(n)=\frac{1}{\sqrt{d_{r d}^{\alpha}}} h_{r d} \mathbf{s}_{r}(n)+\mathbf{w}_{r d}(n),
\end{gathered}
$$

where $\mathbf{w}_{r a}(n) \sim \mathcal{C N}\left(0, N_{0_{r a}} \mathbf{I}_{M}\right)$ and $\mathbf{w}_{r c}(n) \sim$ $\mathcal{C N}\left(0, N_{0_{r c}} \mathbf{I}_{M}\right)$ are the receive antenna and RF to baseband

\footnotetext{
${ }^{1}$ An example of a commercialised power harvester that is able to directly power a battery-less low-power device is that of Powercast's [25].
}

signal conversion AWGNs at $R$, respectively, and $\mathbf{w}_{r d}(n) \sim$ $\mathcal{C N}\left(0, N_{0_{r d}} \mathbf{I}_{M}\right)$ is the overall AWGN due to both the receive antenna and RF to baseband signal conversion at $D$. Furthermore, $\mathbf{s}_{r}(n)$ represents the signal transmitted from $R$ during Phase-2 after normalisation, where the amplification gain is chosen to ensure that the average transmission power at $R$ is fixed to $P_{r}$ and thus, $\mathbf{s}_{r}(n)$ can be expressed as

$$
\mathbf{s}_{r}(n)=\sqrt{\frac{P_{r}}{\frac{1}{d_{s r}^{\alpha}} \kappa P_{s} \sigma_{s r}^{2}+M\left[\kappa N_{0_{r a}}+N_{0_{r c}}\right]}} \mathbf{y}_{s r}(n) .
$$

By substituting (11) in (10), the signal received at $D$ is given as

$$
\begin{aligned}
\mathbf{y}_{r d}(n) & =\underbrace{\frac{\sqrt{\kappa \eta \theta\left|h_{s r}\right|^{2}} P_{s} h_{s r} h_{r d} \mathbf{i}_{m+1}(n)}{\sqrt{d_{s r}^{\alpha} d_{r d}^{\alpha}} \sqrt{\kappa P_{s} \sigma_{s r}^{2}+M d_{s r}^{\alpha} N_{0_{s r}}}}}_{\text {Signal Part }} \\
& +\underbrace{\frac{\sqrt{\eta \theta P_{s}\left|h_{s r}\right|^{2}} h_{r d} \mathbf{w}_{s r}(n)}{\sqrt{d_{r d}^{\alpha}} \sqrt{\kappa P_{s} \sigma_{s r}^{2}+M d_{s r}^{\alpha} N_{0_{s r}}}}+\mathbf{w}_{r d}(n)}_{\text {Noise Part }},
\end{aligned}
$$

where $\mathbf{w}_{s r}(n)=\sqrt{\kappa} \mathbf{w}_{r a}(n)+\mathbf{w}_{r c}(n)$ is the overall noise at $R$ with variance $N_{0_{s r}}=\kappa N_{0_{r a}}+N_{0_{r c}}$. Consequently, the instantaneous received SNR at $D$ can be expressed as

$$
\gamma_{e q}=\frac{a b \rho_{s r} \rho_{r d}\left|h_{s r}\right|^{4}\left|h_{r d}\right|^{2}}{b \rho_{r d}\left|h_{s r}\right|^{2}\left|h_{r d}\right|^{2}+a \rho_{s r} \sigma_{s r}^{2}+M},
$$

where $a=\frac{\kappa}{d_{s r}^{\alpha}}, b=\frac{\eta \theta}{d_{s r}^{\alpha} d_{r d}^{\alpha}}, \rho_{s r}=\frac{P_{s}}{N_{0_{s r}}}$ and $\rho_{r d}=\frac{P_{s}}{N_{0_{r d}}}$. In the aforementioned noncoherent modulation schemes, it is assumed that all waveforms within one symbol interval are equiprobable and have the same energy $P_{s}$.

\section{B. Time Switching-Based Relaying}

The key parameters in the TS relaying protocol for $\mathrm{EH}$ and information processing at $R$ are depicted in Fig. 2a. A certain block of information is transmitted from $S$ to $D$ during a total time of $T$ (sec). Under this protocol, $\beta$ is the portion of the total time in which the relay harvests energy from the source signal, where $0<\beta<1$. Following the same setup as in [6], [15] for TS, the information transmission is performed in the remaining block time, i.e., $(1-\beta) T$, such that half of the fraction of the time, $(1-\beta) T / 2$, denoted as Phase -1 , is used for the source to relay transmission, while the remaining fraction of the time, i.e., $(1-\beta) T / 2$, denoted as Phase- 2 , is used by the relay to forward the information to the destination as shown in Fig. 2b. Based on that, the energy harvested at $R$ is given by

$$
E_{r}=\frac{\eta P_{s}\left|h_{s r}\right|^{2}}{d_{s r}^{\alpha}}(\beta T) .
$$

Based on the fact that $R$ communicates with $D$ for the time $(1-\beta) T / 2$, the transmitted power from the relay node is given by

$$
P_{r}=\frac{E_{r}}{(1-\beta) T / 2}=\frac{2 \eta P_{s}\left|h_{s r}\right|^{2} \beta}{d_{s r}^{\alpha}(1-\beta)} .
$$

In the following, we present the details of the signal model when noncoherent $M$-DPSK and $M$-FSK are employed with TS-based relaying. 


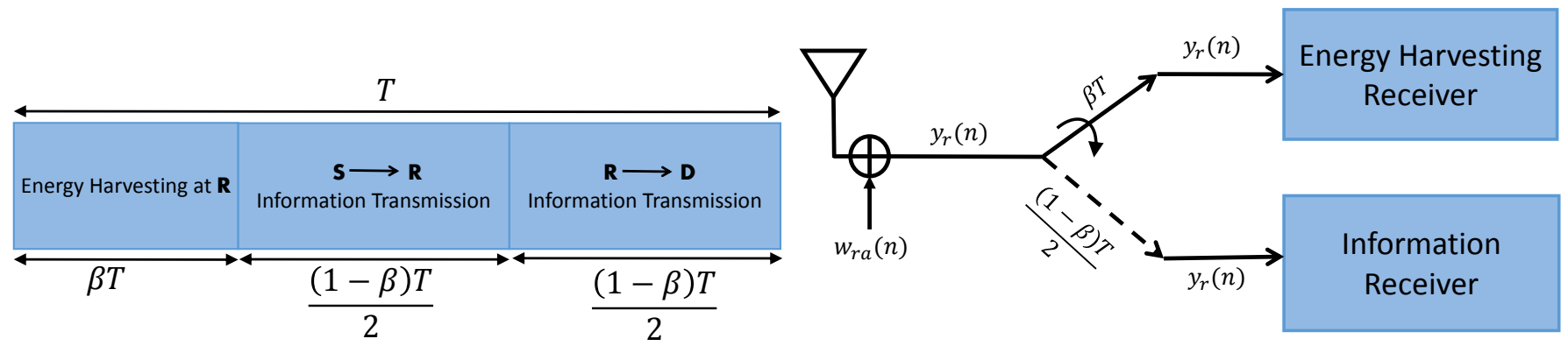

(a)

(b)

Fig. 2: (a) Illustration of the time frame of SWIPT relaying employing the TS protocol (b) Block diagram of the relay receiver architecture with the TS relaying protocol.

1) TS with M-DPSK: At the end of Phase-1, the signal received from $S$ is used solely for information processing at $R$, i.e., no PS is involved and accordingly, the baseband equivalent signal model with $M$-DPSK is given as

$$
\begin{gathered}
y_{s r}(n)=\frac{\sqrt{P_{s}}}{\sqrt{d_{s r}^{\alpha}}} h_{s r} x_{m}(n)+w_{r a}(n)+w_{r c}(n), \\
y_{r d}(n)=\frac{1}{\sqrt{d_{r d}^{\alpha}}} h_{r d} s_{r}(n)+w_{r d}(n),
\end{gathered}
$$

where $w_{r a}(n) \sim \mathcal{C N}\left(0, N_{0_{r a}}\right)$ and $w_{r c}(n) \sim \mathcal{C N}\left(0, N_{0_{r c}}\right)$ are the receive antenna and $\mathrm{RF}$ to baseband signal conversion AWGNs at $R$ and $w_{r d}(n) \sim \mathcal{C N}\left(0, N_{0_{r d}}\right)$ is the overall AWGN due to the receive antenna and RF to baseband signal conversion at $D$, respectively. To ensure that the power of the transmitted signal from $R$ for TS-based relaying with $M$ DPSK is set to $P_{r}$, the signal forwarded from the relay node, $s_{r}(n)$ is given by

$$
s_{r}(n)=\sqrt{\frac{P_{r}}{\frac{1}{d_{s r}^{\alpha}} P_{s} \sigma_{s r}^{2}+N_{0_{r a}}+N_{0_{r c}}}} y_{s r}(n) .
$$

By setting $w_{s r}(n)=w_{r a}(n)+w_{r c}(n), N_{0_{s r}}=N_{0_{r a}}+N_{0_{r c}}$, and upon substituting (18) and (15) into (17), the received signal at $D, y_{r d}(n)$, in terms of $P_{s}, \eta, \beta, d_{s r}^{\alpha}$, and $d_{r d}^{\alpha}$, can be rewritten as

$$
\begin{aligned}
y_{r d}(n) & =\underbrace{\frac{\sqrt{2 \eta \beta\left|h_{s r}\right|^{2}} P_{s} h_{s r} h_{r d} x_{m}(n)}{\sqrt{(1-\beta) d_{s r}^{\alpha} d_{r d}^{\alpha}} \sqrt{P_{s} \sigma_{s r}^{2}+d_{s r}^{\alpha} N_{0_{s r}}}}}_{\text {Signal Part }} \\
+ & \underbrace{\frac{\sqrt{2 \eta \beta P_{s}\left|h_{s r}\right|^{2}} h_{r d} w_{s r}(n)}{\sqrt{(1-\beta) d_{r d}^{\alpha}} \sqrt{P_{s} \sigma_{s r}^{2}+d_{s r}^{\alpha} N_{0_{s r}}}}+w_{r d}(n)}_{\text {Noise Part }} .
\end{aligned}
$$

From (19), the instantaneous received SNR of the relay path, $\gamma_{\text {eq }}$, can be expressed as (8) with $a=\frac{1}{d_{s r}^{\alpha}}, b=\frac{2 \eta \beta}{d_{s r}^{\alpha} d_{r d}^{\alpha}(1-\beta)}$, $\rho_{s r}=\frac{P_{s}}{N_{0_{s r}}}$, and $\rho_{r d}=\frac{P_{s}}{N_{0_{r d}}}$.

2) TS with noncoherent $M-F S K$ : When noncoherent $M$ FSK signaling is employed, the signals received at $R$ at the end of Phase-1 and at $D$ at the end of Phase-2 can be respectively modeled as

$$
\mathbf{y}_{s r}(n)=\frac{\sqrt{P_{s}}}{\sqrt{d_{s r}^{\alpha}}} h_{s r} \mathbf{i}_{m+1}(n)+\mathbf{w}_{r a}(n)+\mathbf{w}_{r c}(n),
$$

and

$$
\mathbf{y}_{r d}(n)=\frac{1}{\sqrt{d_{r d}^{\alpha}}} h_{r d} \mathbf{s}_{r}(n)+\mathbf{w}_{r d}(n),
$$

where $\mathbf{w}_{r a}(n) \sim \mathcal{C N}\left(0, N_{0_{r a}} \mathbf{I}_{M}\right)$ and $\mathbf{w}_{r c}(n) \sim$ $\mathcal{C N}\left(0, N_{0_{r c}} \mathbf{I}_{M}\right)$ are the receive antenna and RF to baseband signal conversion AWGNs at $R$, respectively, and $\mathbf{w}_{r d}(n) \sim$ $\mathcal{C N}\left(0, N_{0_{r d}} \mathbf{I}_{M}\right)$ is the overall AWGNs due to the receive antenna and RF to baseband signal conversion at $D$. The signal received at $R$ in Phase-1 is amplified or scaled to meet an average power constraint $P_{r}$ and thus, the transmitted signal, $\mathbf{s}_{r}(n)$, is specified as

$$
\mathbf{s}_{r}(n)=\sqrt{\frac{P_{r}}{\frac{1}{d_{s r}^{\alpha}} P_{s} \sigma_{s r}^{2}+M\left[N_{0_{r a}}+N_{0_{r c}}\right]}} \mathbf{y}_{s r}(n) .
$$

Combining (15) and (22), followed by a substitution of (22) back into (21), yield the signal received at $D$ as

$$
\begin{aligned}
\mathbf{y}_{r d}(n) & =\underbrace{\frac{\sqrt{2 \eta \beta\left|h_{s r}\right|^{2}} P_{s} h_{s r} h_{r d} \mathbf{i}_{m+1}(n)}{\sqrt{(1-\beta) d_{s r}^{\alpha} d_{r d}^{\alpha}} \sqrt{P_{s} \sigma_{s r}^{2}+M d_{s r}^{\alpha} N_{0_{s r}}}}}_{\text {Signal Part }} \\
& +\underbrace{\frac{\sqrt{2 \eta \beta P_{s}\left|h_{s r}\right|^{2}} h_{r d} \mathbf{w}_{s r}(n)}{\sqrt{(1-\beta) d_{r d}^{\alpha}} \sqrt{P_{s} \sigma_{s r}^{2}+M d_{s r}^{\alpha} N_{0_{s r}}}}+\mathbf{w}_{r d}(n)}_{\text {Noise Part }},
\end{aligned}
$$

where $\mathbf{w}_{s r}(n)=\mathbf{w}_{r a}(n)+\mathbf{w}_{r c}(n)$ is the overall noise at $R$ with variance $N_{0_{s r}}=N_{0_{r a}}+N_{0_{r c}}$. Based on (23), the instantaneous received SNR of the relay link, when TS relaying is employed with noncoherent $M$-FSK, is expressed as (13) with $a=\frac{1}{d_{s r}^{\alpha}}, b=\frac{2 \eta \beta}{d_{s r}^{\alpha} d_{r d}^{\alpha}(1-\beta)}, \rho_{s r}=\frac{P_{s}}{N_{0_{s r}}}$, and $\rho_{r d}=\frac{P_{s}}{N_{0_{r d}}}$.

\section{Ideal Relay Receiver}

For completeness, we investigate in this section the ideal relay receiver. Specifically, when the RF-powered relay is enabled by an ideal receiver, the same received signal is used for information processing and EH [11]. As a result, during Phase-1, the relay node harvests energy and processes the information from the source signal and during Phase-2, it uses the harvested energy to forward the source signal to the destination. Consequently, the available power at the relay due 
TABLE I: Instantaneous Received SNR Expression Parameters for PS, TS, and Ideal Relay Receiver

\begin{tabular}{|l|l|l|l|}
\hline Relaying Protocol & \multicolumn{1}{|c|}{ PS Protocol } & \multicolumn{1}{c|}{ TS Protocol } & \multicolumn{1}{c|}{ Ideal Relay Receiver } \\
\hline$\hat{a}$ & $\hat{a}=\frac{\kappa}{d_{s r}^{\alpha}} \rho_{s r}$ & $\hat{a}=\frac{1}{d_{s r}^{\alpha}} \rho_{s r}$ & $\hat{a}=\frac{1}{d_{s r}^{\alpha}} \rho_{s r}$ \\
\hline$\hat{b}$ & $\hat{b}=\frac{\eta \theta}{d_{s r}^{\alpha} d_{r d}^{\alpha}} \rho_{r d}$ & $\hat{b}=\frac{2 \eta \beta}{d_{s r}^{\alpha} d_{r d}^{\alpha}(1-\beta)} \rho_{r d}$ & $\hat{b}=\frac{\eta}{d_{s r}^{\alpha} d_{r d}^{\alpha}} \rho_{r d}$ \\
\hline$\rho_{s r}$ & $\rho_{s r}=P_{s} / N_{0_{s r}}$ & $\rho_{s r}=P_{s} / N_{0_{s r}}$ & $\rho_{s r}=P_{s} / N_{0_{s r}}$ \\
\hline$\rho_{r d}$ & $\rho_{r d}=P_{s} / N_{0_{r d}}$ & $\rho_{r d}=P_{s} / N_{0_{r d}}$ & $\rho_{r d}=P_{s} / N_{0_{r d}}$ \\
\hline$N_{0_{s r}}$ & $N_{0_{s r}}=\kappa N_{0_{r a}}+N_{0_{r c}}$ & $N_{0_{s r}}=N_{0_{r a}}+N_{0_{r c}}$ & $N_{0_{s r}}=N_{0_{r a}}+N_{0_{r c}}$ \\
\hline
\end{tabular}

to EH is given by $P_{r}=\frac{\eta P_{s}\left|h_{s r}\right|^{2}}{d_{s r}^{\alpha}}$. Using this value of $P_{r}$ in the relay amplification gain and following the same steps as in Sections II-A and II-B, the instantaneous received SNR expression for the ideal relaying receiver can be obtained.

For the convenience of the ensuing analysis, the instantaneous received SNR expressions for the PS, TS, and ideal relay receiver protocols, considering $M$-DPSK and $M$-FSK modulations, can be written in one unified general form as

$$
\gamma_{e q}=\frac{\hat{a} \hat{b}\left|h_{s r}\right|^{4}\left|h_{r d}\right|^{2}}{\hat{b}\left|h_{s r}\right|^{2}\left|h_{r d}\right|^{2}+\hat{a} \sigma_{s r}^{2}+\Psi},
$$

where the constants $\hat{a}$ and $\hat{b}$ differ in the PS, TS, and ideal relay receiver protocols and are defined in Table I. Furthermore, $\Psi$ is set to 1 for $M$-DPSK, and $M$ for $M$-FSK.

Remark 1: Note that the SNR expression in (24) differs from the one in [6] for coherent modulation, which is given by

$$
\gamma_{e q}=\frac{\hat{a} \hat{b}\left|h_{s r}\right|^{4}\left|h_{r d}\right|^{2}}{\hat{b}\left|h_{s r}\right|^{2}\left|h_{r d}\right|^{2}+\hat{a}\left|h_{s r}\right|^{2}+1} .
$$

It is notable that the expression in (24) and the one resulting from coherent modulation as above are different, since the random variable $\left|h_{s r}\right|^{2}$ appears in the second term of the denominator of the latter while it does not in (24). Accordingly, their associated probability density functions (PDFs), comulative distribution functions (CDFs), $n$-th order moments, MGFs, ..etc, are also different, rendering the underlying analysis results for the ASER, outage probability, and throughput to be different.

\section{The Moments And MGF of the Receive SNR}

In this section, we derive an exact closed-form expression for the moments of the receive SNR, $\gamma_{e q}$ in (24), and then employ it to approximate its MGF .

\section{A. Moments of the Receive SNR}

Since deriving the PDF and CDF of the receive SNR expression in (24) is a challenging task, due to the term $\left|h_{s r}\right|^{4}$ in the numerator, we alternatively focus on deriving its moments. The moments, specified as $\mu_{n}=\mathbb{E}\left[\gamma_{\text {eq }}^{n}\right]$, can be derived in the following theorem.

Theorem 1: Assuming that both $S \rightarrow R^{2}$ and $R \rightarrow D$ links undergo independent and identically distributed (i.i.d) Rayleigh fading conditions, the $n^{\text {th }}$-order moment of the

\footnotetext{
${ }^{2} A \rightarrow B$ denotes the link between terminal $A$ and terminal $B$.
}

instantaneous end-to-end SNR of dual-hop AF relaying in noncoherent SWIPT systems is given by

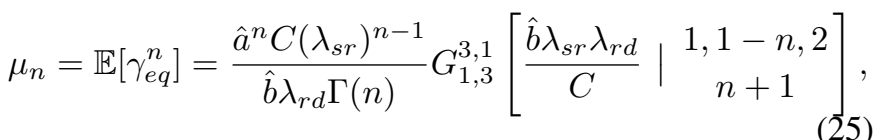

where $G$...[...] is the Meijer G-function as defined in [26, Eq. (8.2.1.1)].

Proof: See Appendix A.

To the best of our knowledge, this result is novel. It is worth noting that (25) is simple and incorporates the Meijer G-function which is a standard built-in function in most of the well-known mathematical software packages, such as MATLAB, MAPLE, and MATHEMATICA, and can therefore, be easily and efficiently evaluated. Theorem 1 turns out to be useful beyond the scope of this paper. Knowing (25), one can establish the MGF, AoF, ASNR, outage probability, achievable throughput, and ASER of the system, as will be presented in the subsequent section. Nonetheless, the result in (25) can be further applied to study other metrics, such as the kurtosis and the skewness that characterise the distribution of the receive SNR and the ergodic capacity of the system.

\section{B. MGF of the Receive SNR}

By definition, the MGF of $\gamma_{e q}$ is $\mathcal{M}_{\gamma_{e q}}(s)=\mathbb{E}\left[e^{-s \gamma_{e q}}\right]$ and can be represented as a formal power series (e.g., Taylor) as

$$
\mathcal{M}_{\gamma_{e q}}(s)=\sum_{n=0}^{\infty} \frac{(-1)^{n}}{n !} \mathbb{E}\left[\gamma_{e q}^{n}\right] s^{n}=\sum_{n=0}^{\infty} \frac{(-1)^{n}}{n !} \mu_{n} s^{n} .
$$

Despite the fact that the moments of all orders, $\mu_{n}$, can be computed in closed-form using the analysis of the preceding subsection, in many cases, we cannot conclude where and whether the power series in (26) is convergent or not. Hence, in practice, only a finite number $W$ can be used. Accordingly, the series in (26) can be truncated as

$$
\mathcal{M}_{\gamma_{e q}}(s)=\sum_{n=0}^{W} \frac{(-1)^{n}}{n !} \mu_{n} s^{n}+\mathcal{O}\left(s^{W+1}\right),
$$

with $\mathcal{O}\left(s^{W+1}\right)$ denoting the terms of order higher than $W$ after the truncation. Having (27) in hand, an approximation to the unknown underlying function $\mathcal{M}_{\gamma_{e q}}(s)$ can be obtained by evaluating only a finite number of the moments. It is demonstrated in [27], [28] that the series, presented in (26), can be efficiently and accurately approximated using the PA method. PA is a well-known method that is applied to approximate infinite power series that are either not guaranteed to converge, converge very slowly or for which a limited number of coefficients is known [27]. The approximation is given in 
terms of a simple closed-form rational function of arbitrary order $X$ for the numerator and arbitrary order $Y$ for the denominator, and whose power series expansion agrees with the $W^{\text {th }}$-order $(W=X+Y)$ power expansion of $\mathcal{M}_{\gamma_{e q}}(s)$ [27]. Consequently, the rational function

$$
P_{[X / Y]}(s) \triangleq \frac{\sum_{i=0}^{X} x_{i} s^{i}}{1+\sum_{i=1}^{Y} y_{i} s^{i}}
$$

is said to be a PA to the series (26), if

$$
P_{[X / Y]}(s) \cong \sum_{n=0}^{W} \frac{(-1)^{n}}{n !} \mu_{n} s^{n}+\mathcal{O}\left(s^{W+1}\right) .
$$

It is straight forward to see from (29) that the moments $\mu_{n}, n=1, \ldots, W$ need to be evaluated to construct the approximant, $P_{[X / Y]}(s)$. Also, the coefficients $x_{i}$ and $y_{i}$ can be easily obtained by matching the coefficients of like powers on both sides. Several issues concerning approximants and the method to determine their coefficients are included in [27], [28]. Padé approximants are available as built-in functions in off-the-shelf mathematical software packages, such as MATLAB, MAPLE, and MATHEMATICA. In this work, we apply the subdiagonal PA, $P_{[X / X+1]}(s)$, to approximate $\mathcal{M}_{\gamma_{e q}}(s)$, since it is only for such order of approximants that the convergence rate and the uniqueness can be assured [28].

\section{Performance Analysis}

In this section we exploit the mathematical tools presented in the previous section to derive a number of important performance measures for SWIPT relay networks employing noncoherent modulation.

\section{A. Receive Average SNR (ASNR)}

The ASNR is the most common and well understood performance measure characterising a digital communication system, owing to its ease of evaluation. It also serves as an excellent indicator of the overall fidelity of the system [29]. The ASNR corresponds to the first moment, $\mu_{1}=\mathbb{E}\left[\gamma_{e q}\right]$, which can be computed by setting $n=1$ in (25).

\section{B. Amount of Fading (AoF)}

For the instantaneous receive SNR, $\gamma_{e q}$, the AoF is defined as [29]

$$
A o F_{\gamma_{e q}}=\frac{\mathbb{E}\left[\gamma_{e q}^{2}\right]}{\left(\mathbb{E}\left[\gamma_{e q}\right]\right)^{2}}-1,
$$

which is obtained by substituting (25) in (30).

\section{Outage Probability}

The outage probability, $P_{\text {out }}$, is another standard performance criterion of wireless systems operating over fading channels. It is defined as the probability that the instantaneous SNR, $\gamma_{e q}$, of the $S \rightarrow R \rightarrow D$ at $D$ falls below a certain predefined threshold, $\gamma_{t h}$, namely,

$$
P_{o u t} \triangleq F_{\gamma_{e q}}\left(\gamma_{t h}\right)=\operatorname{Pr}\left(\gamma_{e q} \leq \gamma_{t h}\right)
$$

where $\gamma_{t h}=2^{R_{T}}-1, R_{T}$ is the transmission rate, and $F_{\gamma_{e q}}($. is the CDF of $\gamma_{e q}$, and can be evaluated by [30]

$$
P_{\text {out }} \triangleq F_{\gamma_{e q}}\left(\gamma_{t h}\right)=\left[\mathcal{L}^{-1}\left(\frac{\mathcal{M}_{\gamma_{e q}}(s)}{s}\right)\right]_{s=\gamma_{t h}},
$$

where $\mathcal{M}_{\gamma_{e q}}(s)$ is the MGF expression pertaining to $\gamma_{s r}$, and $\mathcal{L}^{-1}($.$) denotes the inverse Laplace transform. Herein,$ to evaluate the outage probability, we follow the well-known MGF approach which is achieved by substituting the MGF expression given in (28) into (32), and applying the accurate Euler numerical technique for the inversion of the Laplace transform presented in [30]. Following the steps illustrated therein, the outage probability of the $S \rightarrow R \rightarrow D$ link of noncohernt SWIPT systems can be calculated according to

$$
\begin{aligned}
P_{\text {out }} & =\frac{2^{-Q} e^{A / 2}}{\gamma_{\text {th }}} \sum_{q=0}^{Q}\left(\begin{array}{c}
Q \\
q
\end{array}\right) \sum_{n=0}^{N+q} \frac{(-1)^{n}}{\beta_{n}} \Re\left\{\frac{\mathcal{M}_{\gamma_{e q}}\left(\frac{A+2 \pi j n}{2 \gamma_{t h}}\right)}{\frac{A+2 \pi j n}{2 \gamma_{t h}}}\right\} \\
& +E(A, N, Q),
\end{aligned}
$$

with $\beta_{n}=2$ when $n=0$ and $\beta_{n}=1$ when $n=$ $1,2, \ldots, N$, and $A, Q$ and $N$ denote the truncation parameters. Furthermore, $E(A, N, Q)$ denotes the overall discretisation and truncation error term which can be approximately bounded by [30]

$$
\begin{aligned}
|E(A, N, Q)| & \simeq \frac{e^{-A}}{1-e^{-A}}+\mid \frac{2^{-Q} e^{A / 2}}{\gamma_{t h}} \sum_{q=0}^{Q}(-1)^{N+1+q}\left(\begin{array}{c}
Q \\
q
\end{array}\right) \\
& \times \Re\left\{\frac{\mathcal{M}_{\gamma_{e q}}\left(\frac{A+2 \pi j(N+q+1)}{2 \gamma_{t h}}\right)}{\frac{A+2 \pi j(N+q+1)}{2 \gamma_{t h}}}\right\} \mid .
\end{aligned}
$$

\section{Throughput Analysis}

The throughput, $\tau$, measures the rate of successful information decoding at the destination node, given a fixed transmission rate $R_{T}$, and is defined for the TS and PS relaying protocols as

$$
\tau_{T S}=\frac{\left(1-P_{\text {out }}\right) R_{T}(1-\beta)}{2}
$$

and

$$
\tau_{P S}=\frac{\left(1-P_{\text {out }}\right) R_{T}}{2},
$$

respectively, [6].

\section{E. Average Symbol Error Rate (ASER)}

The aim of this subsection is to analyse the final performance criterion of the underlying SWIPT relaying scheme. By exploiting the PA method described in Section III-B, and the well-known MGF-based unified approach to the ASER analysis for noncoherent and differential modulation over fading channels [31], the ASER of arbitrary $M$-ary noncoherent modulation schemes can be readily evaluated as follows.

The unconditional ASER of the proposed system under $M$ ary FSK modulation scheme is given by [31]

$$
\bar{P}_{s e_{M F S K}}=\sum_{m=1}^{M-1}(-1)^{m+1}\left(\begin{array}{c}
M-1 \\
m
\end{array}\right) \frac{1}{m+1} \mathcal{M}_{\gamma_{e q}}\left(\frac{m}{m+1}\right) \text {. }
$$


When $M$-ary DPSK is employed for SWIPT relaying, the ASER is expressed as [31]

$$
\bar{P}_{s e_{M D P S K}}=\frac{1}{\pi} \int_{0}^{(M-1) \pi / M} \mathcal{M}_{\gamma_{e q}}\left(\frac{g}{1+\sqrt{1-g} \cos \phi}\right) d \phi,
$$

where $g \triangleq \sin ^{2}(\pi / M)$.

Although a closed-form expression cannot be obtained, (38) can be easily computed numerically since it presents a finite-range summation and an integral involving integrands composed of closed-form approximations. By substituting (28) in (37) and (38), the ASER performance of noncoherent $M$ FSK and $M$-DPSK modulation schemes can be evaluated for the desired relaying protocol of any modulation size.

\section{ASYMPTOTIC ANALYSIS}

Although the expressions in (33), (37), and (38) are based on a very tight approximation of the MGF of the receive SNR, they do not provide useful insights into the system performance. Consequently, in this section, we derive further asymptotic expressions which can be used to provide deep insights into the system performance of the considered system model.

Specifically, considering (24), we can alternatively write it as

$$
\gamma_{e q}=\frac{\gamma_{\mathrm{hop}_{1}} \gamma_{\mathrm{hop}_{2}}}{\gamma_{\mathrm{hop}_{2}}+\bar{\gamma}_{\mathrm{hop}_{1}}+\Psi},
$$

where, $\gamma_{\text {hop }_{1}}=\hat{a} \gamma_{s r}$ and $\gamma_{\text {hop }_{2}}=\hat{b} \gamma_{s r} \gamma_{r d}$, and $\bar{\gamma}_{\text {hop }_{1}}=\hat{a} \sigma_{s r}^{2}$. Examining (39), we note that two SNRs, namely, $\gamma_{\mathrm{hop}_{1}}$ and $\gamma_{\text {hop }_{2}}$, parameterise the performance. Allowing the two SNRs to become large does not simplify the analysis further; therefore, we allow only $\gamma_{\text {hop }_{1}}$ to approach infinity, which could be justified when the relay is placed very close to the source. In particular, as $\gamma_{\mathrm{hop}_{1}} \rightarrow \infty^{+}$, (39) can be expressed as

$$
\gamma_{e q} \cong \hat{b} \gamma_{s r}^{2} \gamma_{r d}
$$

which follows by setting $\sigma_{s r}^{2}=1$ for a Rayleigh fading channel.

In the following, we derive asymptotic expressions for the outage probability and the ASER of the system as follows.

\section{A. Asymptotic Outage Probability}

The asymptotic outage probability of (33) is given in the following proposition.

Proposition 1: The asymptotic outage probability of a noncoherent dual-hop SWIPT AF relay network, when the average SNR of the first hop; $S \rightarrow R$, approaches $\infty^{+}$, can be given as

$$
P_{\text {out } 1}^{\infty}=F_{\gamma_{\text {eq }}}^{\infty}(\gamma) \cong 1-\frac{1}{\sqrt{\pi}} G_{3,0}^{0,3}\left[\begin{array}{l|c}
\frac{4 \hat{b} \lambda_{s r}^{2} \lambda_{r d}}{\gamma_{t h}} & 0,0.5,1 \\
-
\end{array}\right] .
$$

\section{Proof: See Appendix B}

By knowing the mathematical behavior of the Meijer Gfunction and recalling that $\hat{b}=\frac{\eta \theta}{d_{s r}^{\alpha} d_{r d}^{\alpha}} \rho_{r d}$ for PS-based relaying and $\hat{b}=\frac{2 \eta \beta}{d_{s r}^{\alpha} d_{r d}^{\alpha}(1-\beta)} \rho_{r d}$ for TS-based relaying, where $\rho_{r d}=\frac{P_{s}}{N_{0_{r d}}}$, we can note from Proposition 1 that as $\hat{b}$ increases, corresponding to increasing the average SNR of the second hop, the second term in (41) increases and the outage probability decreases. This suggests that when the average SNR of the first hop is very high, the outage probability of the system decreases regardless of the PS or TS ratios, i.e., $\theta$ or $\beta$ in PS-based or TS-based relaying, respectively, This will be confirmed in the numerical and simulation results shown in Section VII.

Special Case: when $\hat{b} \rightarrow \infty^{+}$, corresponding to the case when the average SNR of the second hop; $R \rightarrow D$, grows large, we apply the asymptotic representation of the Meijer Gfunction given in [32, Eq. (41)] to derive the outage probability in terms of basic elementary functions as

$$
\begin{aligned}
P_{\text {out } 2}^{\infty} & =F_{\gamma_{e q}}^{\infty}\left(\gamma_{t h}\right) \cong 1-\frac{1}{\sqrt{\pi}}[\underbrace{\frac{1}{\Omega \hat{b}} \Gamma(-0.5)}_{t_{1}} \\
& +\underbrace{\frac{1}{\sqrt{\Omega \hat{b}}} \Gamma(0.5) \Gamma(-0.5)}_{t_{2}}+\Gamma(0.5)],
\end{aligned}
$$

where $\Omega=\left(4 \lambda_{s r}^{2} \lambda_{r d}\right) / \gamma_{t h}$. It is straightforward to notice from (42) that as $\hat{b} \rightarrow \infty^{+}$the term $t_{2}$ dominates over $t_{1}$. In other words, the convergence of $t_{2}$ to zero is slower compared to $t_{1}$, and accordingly, $P_{\text {out } 2}^{\infty}$ can be reduced to

$$
P_{\text {out } 2}^{\infty}=F_{\gamma_{e q}}^{\infty}\left(\gamma_{t h}\right) \cong-\frac{1}{\sqrt{\pi \Omega \hat{b}}} \Gamma(0.5) \Gamma(-0.5) .
$$

We focus now on investigating the diversity order to gain further insights into the impact of $\mathrm{EH}$ on noncoherent relaying systems. Setting $S N R=\hat{b}$, a careful examination of (43) reveals that the outage probability of the considered SWIPT relay system for a given rate, $R_{T}$, for both PS and TS relaying schemes, behaves as $P_{\text {out } 2}^{\infty} \propto S N R^{-\frac{1}{2}}$ at high SNR, which gives rise to a diversity order $d=0.5$ as

$$
d=\lim _{S N R \rightarrow \infty}\left(-\frac{\log P_{\text {out }}}{\log S N R}\right)=-\frac{\log P_{\text {out } 2}^{\infty}}{\log S N R}=0.5 .
$$

It is therefore easy to see from the previous discussion that the obtained diversity order for the considered noncoherent SWIPT system is less than 1 . It can also be concluded that the system performance is limited by the second hop; $R \rightarrow D$, which is the weaker hop, since it is subject to the cascaded fading effect, resulting from $\mathrm{EH}$ at the relay node.

\section{B. Asymptotic ASER}

In order to derive the asymptotic ASER, we utilise the asymptotic CDF, i.e., $F_{\gamma_{e q}}^{\infty}\left(\gamma_{t h}\right)$ obtained in (41) to derive the asymptotic MGF in the following proposition.

Proposition 2: The asymptotic MGF of the approximate receive SNR of a noncoherent dual-hop SWIPT AF relay network when the average SNR of the first hop, $S \rightarrow R$, approaches $\infty^{+}$, can be given as

$$
\mathcal{M}_{\gamma_{e q} 1}^{\infty}(s) \cong 1-\frac{1}{\sqrt{\pi}} G_{3,1}^{1,3}\left[\begin{array}{ll|c}
4 \hat{b} \lambda_{s r}^{2} \lambda_{r d} s & 0,0.5,1 \\
1
\end{array}\right] .
$$




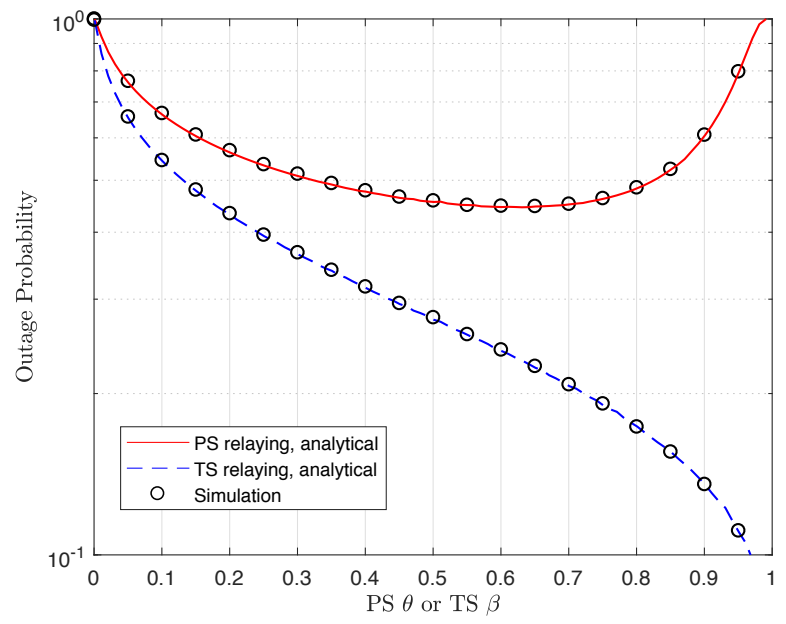

Fig. 3: Outage probability, $P_{\text {out }}$, with respect to $\beta$ for the TS protocol and $\theta$ for the PS protocol for SNR $=20 \mathrm{~dB} . \eta=1, P_{s}=1$, and $d_{s r}=d_{r d}=1$.

Proof: The proof follows by recalling the definition of the MGF, $\mathcal{M}_{\gamma_{e q}}(s)=\mathbb{E}\left[e^{-s \gamma_{e q}}\right]$, then using integration by parts to express the MGF in terms of the CDF as

$$
\mathcal{M}_{\gamma_{e q}}(s)=s \int_{0}^{\infty} e^{-s \gamma} F_{\gamma_{e q}}(\gamma) d \gamma
$$

By substituting the asymptotic CDF of (41) into (46), then applying the transformation [26, Eq. (8.2.2.14)], followed by some mathematical manipulations and finally with the aid of [33, Eq. (7.813.2)], the desired result is obtained.

Special Case: when $\hat{b} \rightarrow \infty^{+}$, corresponding to the scenario when the average SNR of the second hop; $R \rightarrow D$, grows large, we apply the asymptotic representation of the Meijer G-function given in [32, Eq. (41)] to derive the MGF in terms of basic elementary functions as

$$
\begin{aligned}
\mathcal{M}_{\gamma_{e q} 2}^{\infty}(s) \cong 1-\frac{1}{\sqrt{\pi}}\left[\frac{1}{\xi \hat{b} s} \Gamma(-0.5)\right. \\
\left.+\frac{1}{\sqrt{\xi \hat{b} s}} \Gamma(0.5) \Gamma(-0.5) \Gamma(1.5)+\Gamma(0.5)\right],
\end{aligned}
$$

where $\xi=4 \lambda_{s r}^{2} \lambda_{r d}$. Similar to (42), (47) can be further reduced to

$$
\mathcal{M}_{\gamma_{e q} 2}^{\infty}(s) \cong-\frac{1}{\sqrt{\pi \xi \hat{b} s}} \Gamma(0.5) \Gamma(-0.5) \Gamma(1.5) .
$$

Capitalising on the previously derived asymptotic MGF expressions, the evaluation of the ASER of $M$-ary FSK and $M$-ary DPSK is discussed in the following Corollary.

Corollary 1: The asymptotic ASER of $M$-ary FSK and $M$ ary DPSK of noncoherent SWIPT relaying systems can be evaluated by substituting (45) or (48) in (37) and (38), respectively. For the special cases of binary FSK (BFSK) and BDPSK, the asymptotic ASERs can be obtained in closed-forms by substituting either (45) or (48) in $\bar{P}_{\text {se }_{B F S K}}^{\infty} \cong 0.5 \mathcal{M}_{\gamma_{\text {eq }}}^{\infty}(0.5)$ and $\bar{P}_{s e_{B D P S K}}^{\infty} \cong 0.5 \mathcal{M}_{\gamma_{e q}}^{\infty}(1)$, respectively [31].

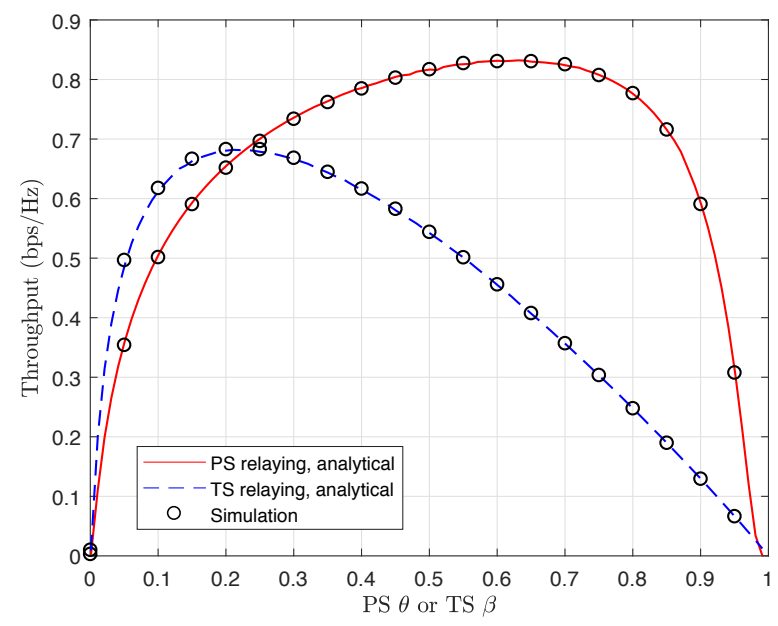

Fig. 4: Throughput, $\tau$, at $D$ with respect to $\beta$ for the TS protocol and $\theta$ for the PS protocol for SNR $=20 \mathrm{~dB} \cdot \eta=1, P_{s}=1$, and $d_{s r}=d_{r d}=1$.

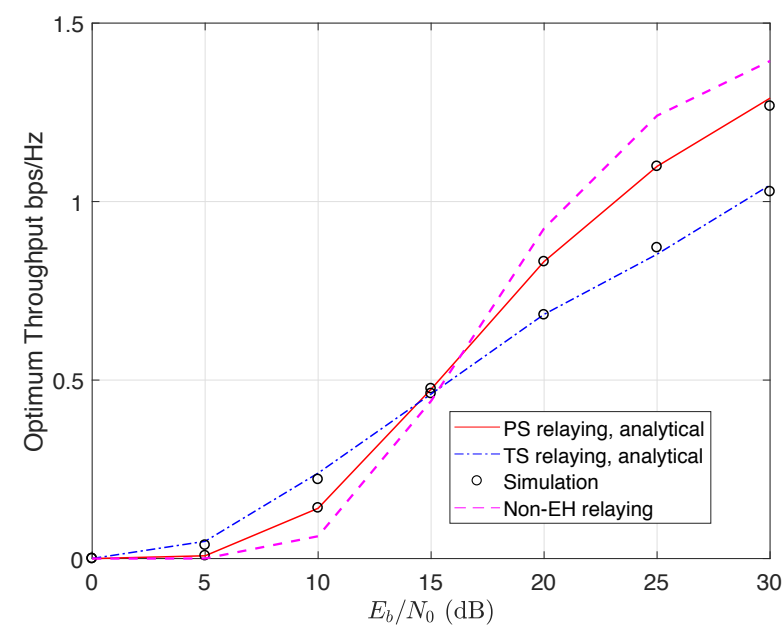

Fig. 5: Optimal throughput, $\tau$, for the TS and PS protocols with respect to SNR. $\eta=1, P_{s}=1, R_{T}=3 \mathrm{bps} / \mathrm{Hz}$, and $d_{s r}=d_{r d}=1$.

Proposition 2 and Corollary 1 reveals that as $\hat{b}$ increases, corresponding to increasing the average SNR of the second hop, the second term in (45) increases and $\bar{P}_{s e_{B F S K}}^{\infty}$ and $\bar{P}_{s e_{B D P S K}}^{\infty}$ decrease. This suggests that when the average SNR of the first hop is very high, the ASER of the system decreases regardless of the PS or TS ratios, i.e., $\theta$ or $\beta$ in PS-based or TS-based relaying, respectively.

\section{NumERicAl AND Simulation RESUlts}

In this section, we provide numerical and simulation results to illustrate and validate the accuracy of the proposed analytical framework.

Unless otherwise stated, we set the source transmission rate to $R_{T}=3 \mathrm{bits} / \mathrm{sec} / \mathrm{Hz}$, the $\mathrm{EH}$ efficiency $\eta=1$, the source transmission power $P_{s}=1$ Watt and path loss $\alpha=2.7$ [6]. We assume that $N_{0} \triangleq N_{0_{s r}}=N_{0_{r d}}$ and for simplicity, we assume that the antenna noise and information receiver circuit noise at the relay node have equal variances, i.e., $N_{0_{r a}}=$ 


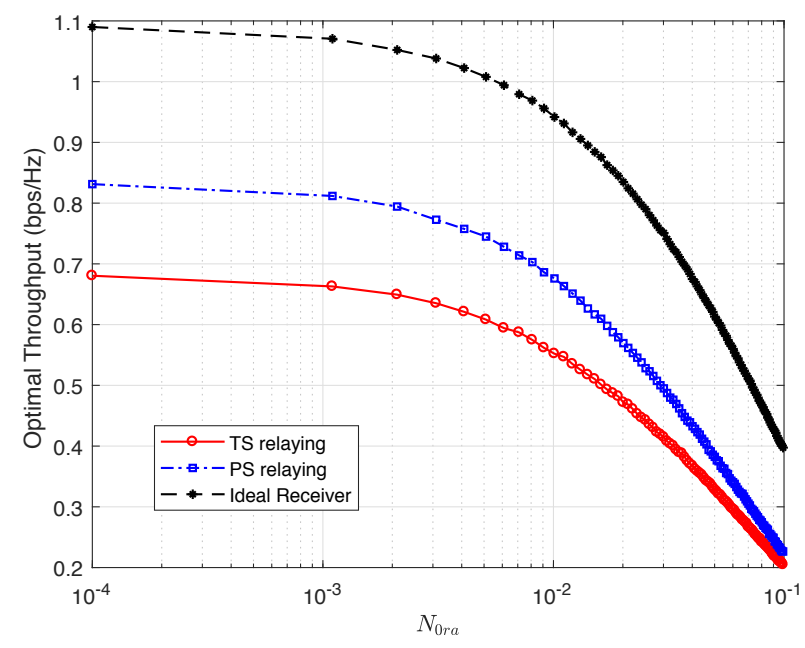

(a)

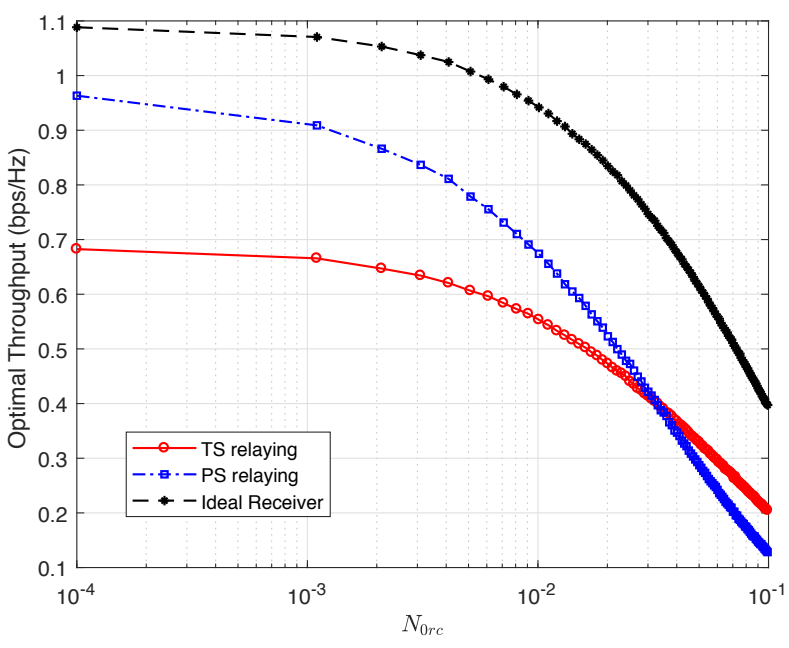

(b)

Fig. 6: Optimal throughput for the ideal receiver, the TR and the PR protocols for (a) different values of antenna noise variance $N_{0_{r a}}$ and $N_{0_{r c}}=0.01$ (fixed) and (b) different values of conversion noise variance $N_{0_{r c}}$ and $N_{0_{r a}}=0.01$ (fixed). Other parameters: $R_{T}=3 \mathrm{bps} / \mathrm{Hz}$, $P_{s}=1, \eta=1$, and $d_{s r}=d_{r d}=1$.

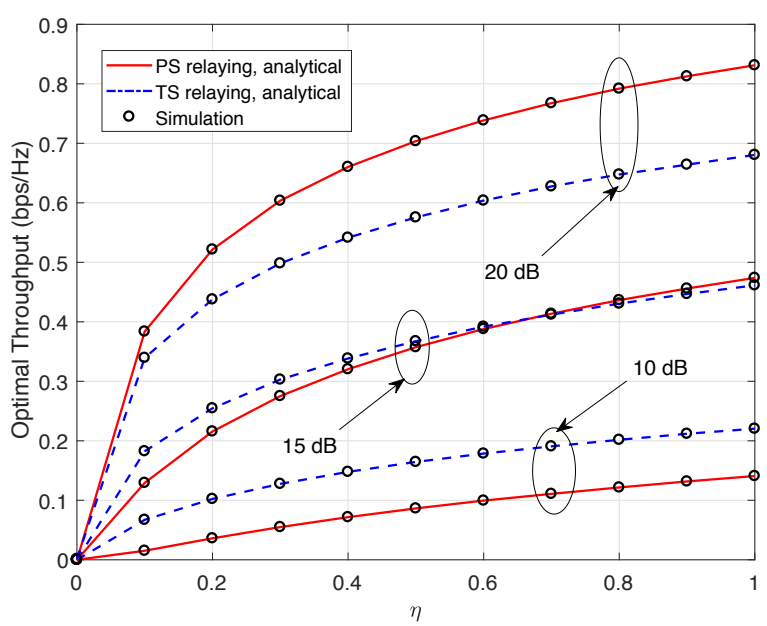

Fig. 7: Optimal throughput, $\tau$, with respect to the EH efficiency, $\eta$, for the TS and PS protocols, respectively, at SNR $=10,15$, and 20 dB. $P_{s}=1, R_{T}=3 \mathrm{bps} / \mathrm{Hz}$, and $d_{s r}=d_{r d}=1$.

$N_{0_{r c}} \triangleq N_{0} / 2$. Since the only energy supplied to the whole network is the transmit power, $P_{s}$, applied to the source, the performance of the whole system is parameterised by SNR $\triangleq$ $P_{s} / N_{0}$. Furthermore, the distances $d_{s r}$ and $d_{r d}$ are normalised to unity. Additionally, the mean values, $\lambda_{s r}$ and $\lambda_{r d}$, of the exponential random variables $\gamma_{s r}$ and $\gamma_{r d}$, respectively, are set to 1 .

Following the parameter values used in [30], in our simulation results, we consider $A=23$ and parameters $Q$ and $N$ are set to 15 and 21 , respectively, so as to ensure a discretisation error that is less than $10^{-10}$. However, the overall resulting error is negligible compared to the actual outage probability value.

In Figs. 3 and 4, we analyse the impact of $\beta$ and $\theta$ on the outage probability, $P_{\text {out }}$, and the achievable throughput, $\tau$, of the system, when the SNR is fixed to $20 \mathrm{~dB}$. As it can be seen from Figs. 3 and 4, there is a perfect match between the simulation and the analytical results for the entire range of the values of $\beta$ or $\theta$, indicating the high accuracy of the PA method applied to approximate the MGF of the receive SNR. It can be also observed that unlike the TS protocol, the PS protocol has an optimal value for $\theta$ that minimises the outage probability. While Fig. 4 demonstrates that there exists an optimal value for $\beta$ and $\theta$ maximising the throughput. This is because, for the PS protocol, as $\theta$ increases from 0 to an optimal value, $(\theta=0.631)$, more power is exploited for $\mathrm{EH}$ and consequently, the relay node transmits with a higher power. Therefore, the outage probability is reduced and, yielding higher values of throughput to be observed at the destination. However, as $\theta$ increases from its optimal value, more power is consumed on $\mathrm{EH}$ and less power is left for source to relay information processing. As a result of weak signal strength received at the relay, the relay amplifies the noisy signal and forwards it to the destination, leading to a higher outage probability and smaller throughput to occur at the destination node. On the contrary, as $\beta$ increases from 0 to 1 for the TS protocol, more time is spent on $\mathrm{EH}$ and, thus, higher power is available at the relay and less outage probability is observed at the destination node as a result of a higher receive SNR. However, the performance of the system in terms of the throughput has a similar trend to that marked for the PS protocol. In particular, as $\beta$ increases from 0 to an optimal value ( $\beta=0.221)$, more time is allocated for $\mathrm{EH}$ causing higher power to be available for information relaying and, thus, higher throughput is observed at the destination node. On the other hand, as $\beta$ increases above its optimal value, less time is available for information transmission due to a smaller value of $(1-\beta) / 2$, and as a consequence, the throughput observed at the destination node decreases.

Remark 2: In order to carry out a fair performance comparison between the TS and the PS protocols, it is desirable to 


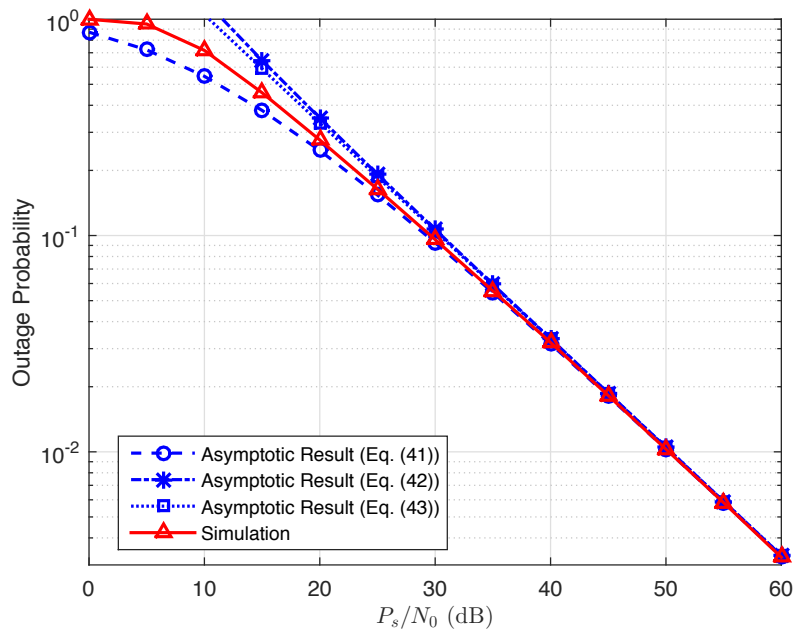

(a) TS Protocol

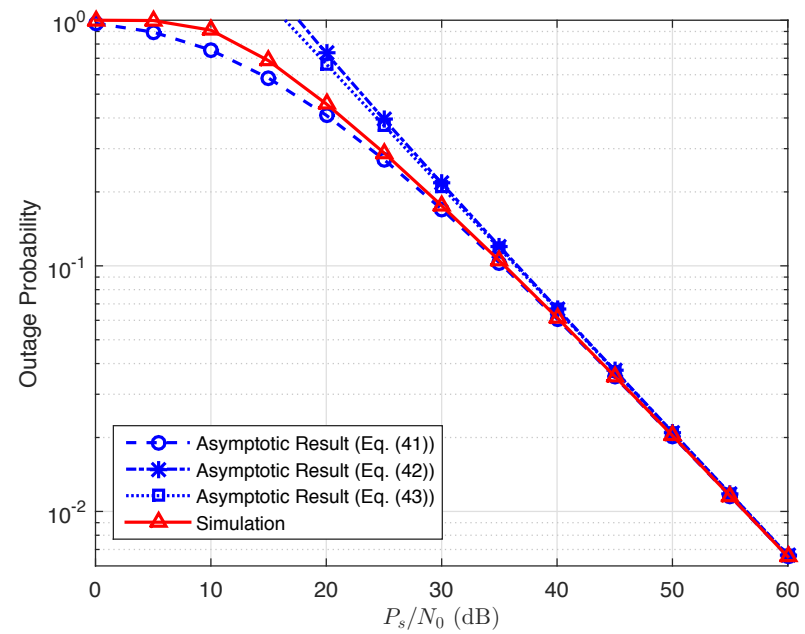

(b) PS Protocol

Fig. 8: Outage probability asymptotic results with respect to SNR, $\eta=1, P_{s}=1, R_{T}=3 \mathrm{bps} / \mathrm{Hz}$, and $d_{s r}=d_{r d}=1$.

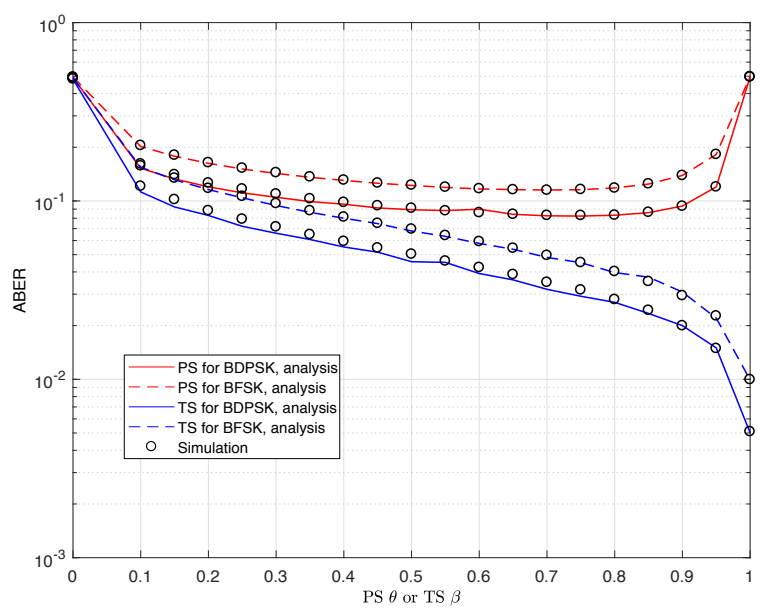

Fig. 9: ASER with respect to $\beta$ or $\theta$ for TS or PS protocols, respectively, employing BDPSK and BFSK at SNR $=20 \mathrm{~dB} \cdot \eta=1$, $P_{s}=1, R_{T}=3 \mathrm{bps} / \mathrm{Hz}$, and $d_{s r}=d_{r d}=1$.

find the values of $\beta$ and $\theta$ that maximise/minimise the value of the performance metric, i.e., throughput or ASER. Due to the $P A$ approximation of the MGF of the receive SNR, the task of finding closed-form expressions for the optimal values of $\beta$ and $\theta$ seems intractable. Nevertheless, the optimisation can be done offline by numerically computing the optimal values of $\beta$ and $\theta$ that maximise/minimise the value of the throughput/ASER, for certain given system parameters, including, EH efficiency $\eta$, source transmission rate $R_{T}$, source power $P_{s}$, $S \rightarrow R$ distance $d_{s r}, R \rightarrow D$ distance, $d_{r d}$, and SNR value.

Fig. 5, we examine the optimal throughput, $\tau$, for different values of SNR such that the performance gain of one relaying scheme over the other can be quantified at any target throughput. Furthermore, the conventional grid-powered non-
EH relaying system is plot as a benchmark ${ }^{3}$. Fig. 5 shows that at lower SNR values (from 0 to $15 \mathrm{~dB}$ ) both TS and PS schemes outperform the the conventional non-EH AF relaying protocol, assuming that noncoherent modulation is applied. This stems from the fact that the SWIPT relaying system is able to boost its overall performance over the nonEH system by allowing the relay to harness extra energy from the source RF signals while accounting for the energyrate tradeoff. However, it can be also noticed that the nonEH relaying scheme outperforms the two SWIPT relaying protocols as the SNR exceeds $15 \mathrm{~dB}$. This is due to the effect of cascaded fading resulting from instantaneous EH at the relay as shown in the numerator of the receive SNR expression in (24). Comparing the TS and the PS protocols, Fig. 5 illustrates that the TS protocol is superior to the PS protocol in achieving higher values of the throughput in the low SNR regime. This result is consistent with the one presented in [6] for coherent modulation in SWIPT relaying systems, where the same instantaneous EH assumption is considered for a single relay.

Fig. 6 plots the optimal throughput for the ideal relay receiver, the TS and the PS protocols for different values of antenna noise variance, $N_{0_{r a}}$ (see Fig. 6a for fixed $N_{0_{r c}}=0.01$ ) and different values of conversion noise variance, $N_{0_{r c}}$ (see Fig. $6 \mathrm{~b}$ for fixed $N_{0_{r a}}=0.01$ ). As expected, the performance of the ideal receiver outperforms the TS and PS protocols for different values of the noise variances, since it is based on the assumption of processing information and extracting power from the same received signal. It is interesting to note that the throughput performance gap between the TS protocol and the ideal receiver becomes constant, as noise variances, $N_{0_{\text {ra }}}$ or $N_{0_{r c}}$ approach 0 . On the other hand, Fig. 6b shows that the throughput performance gap between the PS protocol and

\footnotetext{
${ }^{3}$ The powers applied to a conventional relay non-EH system are $P_{s} / 2$ at $S$ and $P_{s} / 2$ at $R$. This ensures a fair comparison with the EH system and that the total energy supply of both networks remains the same and equal to that of the noncooperative non-EH scheme.
} 


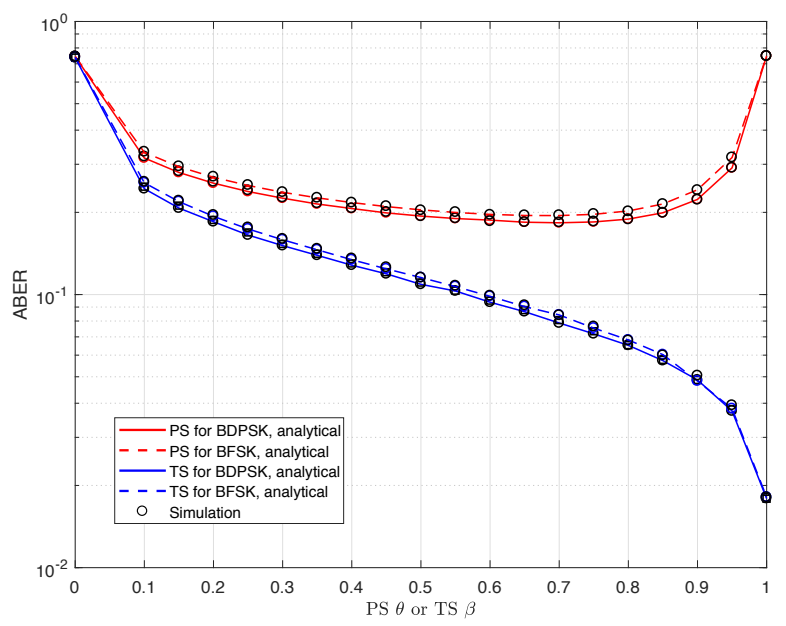

(a)

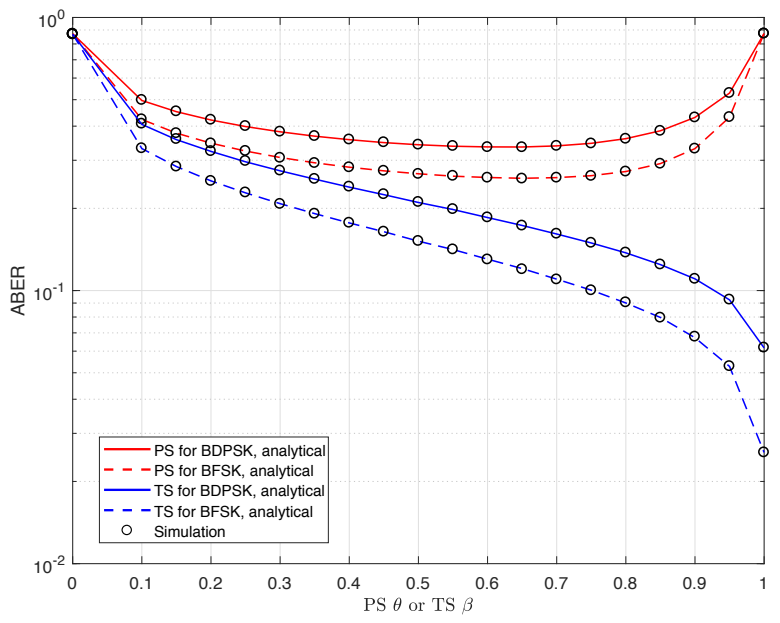

(b)

Fig. 10: (a) ASER with respect to $\beta$ or $\theta$ for TS or PS protocols, respectively, employing 4-DPSK and 4-FSK. (b) ASER with respect to $\beta$ or $\theta$ for TS or PS protocols, respectively, employing 8 -DPSK and 8 -FSK. For both (a) and (b) $\mathrm{SNR}=20 \mathrm{~dB}, \eta=1, P_{s}=1, R_{T}=3$ $\mathrm{bps} / \mathrm{Hz}$, and $d_{s r}=d_{r d}=1$.

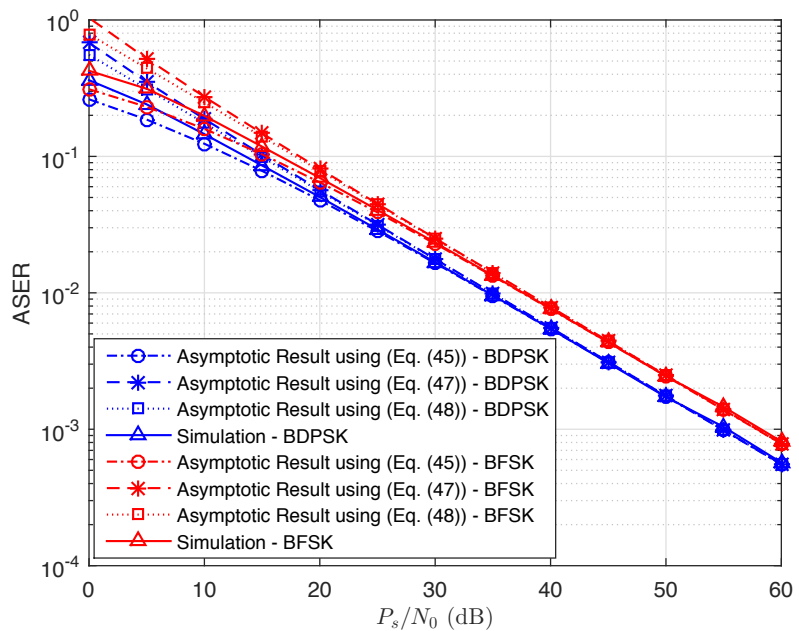

(a) TS Protocol

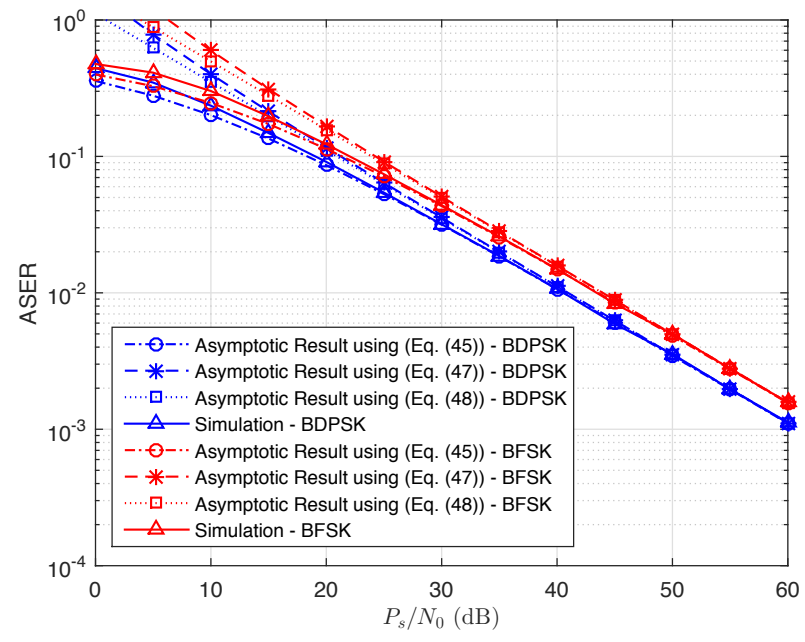

(b) PS Protocol

Fig. 11: ASER asymptotic results with respect to SNR, $\eta=1, P_{s}=1, R_{T}=3 \mathrm{bps} / \mathrm{Hz}$, and $d_{s r}=d_{r d}=1$.

the ideal receiver decreases, as conversion noise variance $N_{0_{r c}}$ approaches 0 .

Fig. 7 presents the optimal throughput, $\tau$, for both the TS and the PS protocols with respect to the EH efficiency, $\eta$, for three different values of the SNR, i.e., $\mathrm{SNR}=10,15$, and 20 $\mathrm{dB}$. It can be observed that at a high SNR value $(\mathrm{SNR}=20$ $\mathrm{dB}$ ), the PS protocol outperforms the TS protocol for the entire range, while it is vice versa at a low SNR value $(\mathrm{SNR}=10$ $\mathrm{dB}$ ). However, at $\mathrm{SNR}=15 \mathrm{~dB}$, which is the intersection point shown in Fig. 5, the TS protocol outperforms the PS protocol when $\eta \leq 0.6$ and as $\eta$ increases beyond 0.6 , the throughput performance gap between the two protocols becomes almost insignificant.

In Fig. 8, we plot the asymptotic outage probability results derived in (41), (42), and (43) against the SNR, for both the TS $(\beta=0.5)$ and PS $(\theta=0.5)$ relaying protocols. It is observed that all three asymptotic results perfectly match the simulation results in the high SNR regime, proving their high accuracy and significant simplicity in computing the outage probability in this region.

Fig. 9 presents the ASER performance of BDPSK and binary FSK (BFSK) vs. $\beta$ and $\theta$ for the TS and the PS protocols, respectively, while fixing the SNR at $20 \mathrm{~dB}$. The analytical results are obtained by computing (38) and (37) for BDPSK and BFSK, respectively $(M=2)$. Several important performance insights can be extracted from Fig. 9. First, it is observed that the TS protocol outperforms the PS protocol across the entire range of $\beta$ or $\theta$ values. Second, it can be noticed that for binary signaling, the performance of the noncoherent BFSK scheme is inferior to the BDPSK scheme for both TS and PS protocols. Third, Fig. 9 demonstrates that there exists a unique optimum value for the PS protocol at 
which the ASER value is minimised, while this is not the case for the TS protocol.

To examine the impact of higher constellation size $M>2$ on the ASER performance of the adopted noncoherent $M$ DPSK and $M$-FSK signalings, we depict in Figs. 10a and $10 \mathrm{~b}$, the ASER performance of the TS and PS protocols for 4-DPSK and 4-FSK, and 8-DPSK and 8-FSK, respectively, when the SNR is fixed to $20 \mathrm{~dB}$. For the case of $M=4$, Fig. 10a illustrates that 4-DPSK slightly outperforms 4-FSK, irrespective of the SWIPT relaying protocol, i.e., TS or PS. As $M$ is increased from 4 to 8 , it can be observed from Fig. 10b that 8-FSK significantly outperforms 8-DPSK, irrespective of the SWIPT relaying protocol. The preceding results suggest that when $M \geq 8, M$-FSK becomes more energy efficient in terms of energy consumption than $M$-DPSK. This is also reported in [15].

Finally, in Fig. 11 we present the asymptotic ASER results derived using the MGF asymptotic expressions in (45), (47), and (46) with respect to the SNR for the TS $(\beta=0.5)$ and PS $(\theta=0.5)$ relaying protocols considering BFSK and BDPSK. It is shown that all asymptotic results fully coincide with the simulation results in the high SNR regime. This verifies the accuracy and efficiency of these results in quantifying the ASER performance of the system in this region.

\section{CONCLUSIONS}

In this paper, we presented analytical expressions for the moments and the MGF of the end-to-end SNR of a noncoherent SWIPT dual-hop relay system, adopting the TS or the PS as the receiver architecture at the relay node. Capitalising on these expressions, we proposed new unified formulas for various performance metrics, including the outage probability, achievable throughput, and ASER of two noncoherent modulation schemes. Further, we derived and presented novel asymptotic expressions for the outage probability and ASER. Additionally, we analytically demonstrated that the diversity order of the considered system is less than 1. Furthermore, the offered unified asymptotic results are applicable beyond the scope of this paper and opens the door for simple further analysis of a wide array of other scenarios and performance metrics, such as ergodic capacity. The analytical model was corroborated with computer simulations. Our results demonstrated that there is a unique value for the PS ratio that minimises the outage probability of the system, while such a value does not exist for the TS protocol. We also showed that, considering the system throughput, the TS relaying scheme is superior to the PS relaying scheme at lower SNR values.

\section{APPENDIX A}

PROOF OF THEOREM I

Let $\gamma_{s r}=\left|h_{s r}\right|^{2}, \gamma_{r d}=\left|h_{r d}\right|^{2}, C=\hat{a} \sigma_{s r}^{2}+\Psi$. The $n^{\text {th }}$ order moment of the receive SNR in (24) can be calculated as $(49 \mathrm{e})$ at the top of this page, where $\Gamma($.$) is the Gamma$ function [33, Eq. (8.310.1)]. Hereby, (49b) follows because $f_{\gamma_{s r}}\left(\gamma_{s r}\right)$ and $f_{\gamma_{r d}}\left(\gamma_{r d}\right)$ are the PDFs of the exponential random variables $\gamma_{s r}$ and $\gamma_{r d}$, with means $\lambda_{s r}$ and $\lambda_{r d}$, respectively. Furthermore, (49d) is obtained by expressing the integrands of $I_{1}$ in (49c) in terms of their Meijer G-function representations. Specifically, the second integrand of $I_{1}$ can be expressed as $\frac{1}{\Gamma(n)} G_{1,1}^{1,1}\left[\frac{\hat{b} \gamma_{s r} \gamma_{r d}}{C} \mid \begin{array}{l}1 \\ n\end{array}\right]$, where the equality in [26, Eq. (8.4.2.5)] is used followed by applying the transformation [26, Eq. (8.2.2.14)]. Also, the third integrand is rewritten by making use of the equality $e^{-\frac{\gamma_{s r}}{\lambda_{s r}}}=G_{0,1}^{1,0}\left[\frac{\gamma_{s r}}{\lambda_{s r}} \mid \overline{0}\right]$ [26, Eq. (8.4.3.1)]. Then, by exploiting the integral identity [26, Eq. (2.24.1.1)] followed by performing some algebraic manipulations, $I_{1}$ can be derived in a closed-form as in $(49 \mathrm{e})$. Finally, by expressing $e^{-\frac{\gamma_{r d}}{\lambda_{r d}}}=G_{0,1}^{1,0}\left[\frac{\gamma_{r d}}{\lambda_{r d}} \mid \begin{array}{c}- \\ 0\end{array}\right]$ and using again the aid of [26, Eq. (2.24.1.1)], the desired result in (25) is reached.

$$
\begin{aligned}
& \mu_{n}=\int_{0}^{\infty} \int_{0}^{\infty}\left(\frac{\hat{a} \hat{b} \gamma_{s r}^{2} \gamma_{r d}}{\hat{b} \gamma_{s r} \gamma_{r d}+C}\right)^{n} f_{\gamma_{s r}}\left(\gamma_{s r}\right) f_{\gamma_{r d}}\left(\gamma_{r d}\right) d \gamma_{s r} d \gamma_{r d} \\
& =\frac{1}{\lambda_{s r} \lambda_{r d}} \int_{0}^{\infty} \underbrace{\int_{0}^{\infty}\left(\frac{\hat{a} \hat{b} \gamma_{s r}^{2} \gamma_{r d}}{\hat{b} \gamma_{s r} \gamma_{r d}+C}\right)^{n} \exp \left(-\frac{\gamma_{s r}}{\lambda_{s r}}\right) d \gamma_{s r}}_{I_{1}} \exp \left(-\frac{\gamma_{r d}}{\lambda_{r d}}\right) d \gamma_{r d} \\
& =\frac{\hat{a}^{n}}{\lambda_{s r} \lambda_{r d}} \int_{0}^{\infty} \underbrace{\int_{0}^{\infty} \gamma_{s r}^{n}\left(1+\frac{C}{\hat{b} \gamma_{s r} \gamma_{r d}}\right)^{-n} \exp \left(-\frac{\gamma_{s r}}{\lambda_{s r}}\right) d \gamma_{s r}}_{I_{1}} \exp \left(-\frac{\gamma_{r d}}{\lambda_{r d}}\right) d \gamma_{r d} \\
& =\frac{\hat{a}^{n}}{\lambda_{s r} \lambda_{r d} \Gamma(n)} \int_{0}^{\infty} \underbrace{\int_{0}^{\infty} \gamma_{s r}^{n} G_{1,1}^{1,1}\left[\left.\frac{\hat{b} \gamma_{s r} \gamma_{r d}}{C}\right|_{n} ^{1} G_{0,1}^{1,0}\left[\left.\frac{\gamma_{s r}}{\lambda_{s r}}\right|_{0} ^{-}\right] d \gamma_{s r}\right.}_{I_{1}} \exp \left(-\frac{\gamma_{r d}}{\lambda_{r d}}\right) d \gamma_{r d} \\
& =\frac{\hat{a}^{n}}{\lambda_{s r} \lambda_{r d} \Gamma(n)}\left(\frac{\hat{b}}{C}\right)^{-n-1} \int_{0}^{\infty} \gamma_{r d}^{-n-1} G_{2,1}^{1,2}\left[\begin{array}{l|l}
\frac{\hat{b} \lambda_{s r} \gamma_{r d}}{C} & 1, n+2 \\
2 n+1
\end{array}\right] \exp \left(-\frac{\gamma_{r d}}{\lambda_{r d}}\right) d \gamma_{r d} .
\end{aligned}
$$




\section{APPENDIX B}

\section{PROOF OF PROPOSITION 1}

The outage probability of the approximated $\gamma_{e q}$ can be expressed as

$$
\begin{aligned}
P_{\text {out } 1}^{\infty} & =\operatorname{Pr}\left(\hat{b} \gamma_{s r}^{2} \gamma_{r d} \leq \gamma_{t h}\right)=\operatorname{Pr}\left(\gamma_{r d} \leq \frac{\gamma_{t h}}{\hat{b} \gamma_{s r}^{2}}\right) \\
& =\int_{0}^{\infty} f_{\gamma_{s r}}\left(\gamma_{s r}\right)\left(1-\exp \left(-\frac{\gamma_{t h}}{\hat{b} \lambda_{r d} \gamma_{s r}^{2}}\right)\right) d \gamma_{s r} \\
& =1-\frac{1}{\lambda_{s r}} \int_{0}^{\infty} \exp \left(-\frac{\gamma_{s r}}{\lambda_{s r}}\right) \exp \left(-\frac{\gamma_{t h}}{\hat{b} \lambda_{r d} \gamma_{s r}^{2}}\right) d \gamma_{s r} \\
& =1-\frac{1}{\lambda_{s r}} \int_{0}^{\infty} \exp \left(-\frac{\gamma_{s r}}{\lambda_{s r}}\right) G_{1,0}^{0,1}\left[\left.\frac{\hat{b} \lambda_{r d} \gamma_{s r}^{2}}{\gamma_{t h}}\right|_{-} ^{1}\right] d \gamma_{s r},
\end{aligned}
$$

where (50c) follows from (50b) because $f_{\gamma_{s r}}\left(\gamma_{s r}\right)$ is the PDF of the exponential random variable $\gamma_{s r}$. Moreover, (50d) is obtained by using the identity [26, Eq. (8.4.3.1)] to express the second integrand of (50c) in terms of its Meijer G-function representation whose arguments are then inverted by applying the transformation [26, Eq. (8.2.2.14)]. Finally, by making use of the identity in [33, Eq. (7.813.2)], we derive the desired result in (41).

\section{REFERENCES}

[1] L. Mohjazi, S. Muhaidat, M. Dianati, and M. Al-Qutayri, "Outage probability and throughput of SWIPT relay networks with differential modulation," in Proc. IEEE 86th Conf. Veh. Technol. Conf, (VTC-Fall), Toronto, Canada, Sept. 2017, pp. 1-6.

[2] S. Bi, C. K. Ho, and R. Zhang, "Wireless powered communication: opportunities and challenges," IEEE Commun. Mag., vol. 53, no. 4, pp. 117-125, Apr. 2015.

[3] L. Mohjazi, M. Dianati, G. Karagiannidis, S. Muhaidat, and M. AlQutayri, "RF-powered cognitive radio networks: technical challenges and limitations," IEEE Commun. Mag., vol. 53, no. 4, pp. 94-100, Apr. 2015.

[4] L. Mohjazi, I. Ahmed, S. Muhaidat, M. Dianati, and M. Al-Qutayri, "Downlink beamforming for SWIPT multi-user MISO underlay cognitive radio networks," IEEE Commun. Lett., vol. 21, no. 2, pp. 434-437, Oct. 2017.

[5] L. R. Varshney, "Transporting information and energy simultaneously," in Proc. IEEE Int. Symp. Inf. Theory, Toronto, Canada, July 2008, pp. $1612-1616$.

[6] A. Nasir, X. Zhou, S. Durrani, and R. Kennedy, "Relaying protocols for wireless energy harvesting and information processing," IEEE Trans. Wireless Commun., vol. 12, no. 7, pp. 3622-3636, July 2013.

[7] K. M. Rabie, B. Adebisi, and M. S. Alouini, "Half-duplex and fullduplex AF and DF relaying with energy-harvesting in Log-Normal fading," IEEE Trans. Green Commun. Netw., vol. 1, no. 4, pp. 468480, Dec. 2017.

[8] X. Zhou and Q. Li, "Energy efficiency optimisation for SWIPT AF twoway relay networks," Electron. Lett., vol. 53, no. 6, pp. 436-438, Mar. 2017.

[9] T. Li, P. Fan, and K. B. Letaief, "Outage probability of energy harvesting relay-aided cooperative networks over rayleigh fading channel," IEEE Trans. Veh. Technol., vol. 65, no. 2, pp. 972-978, Feb. 2016.

[10] H. Lee, C. Song, S. H. Choi, and I. Lee, "Outage probability analysis and power splitter designs for SWIPT relaying systems with direct link," IEEE Commun. Lett., vol. 21, no. 3, pp. 648-651, Nov. 2017.

[11] X. Zhou, R. Zhang, and C. K. Ho, "Wireless information and power transfer: Architecture design and rate-energy tradeoff," IEEE Trans. Commun., vol. 61, no. 11, pp. 4754-4767, Nov. 2013.
[12] T. D. P. Perera, D. N. K. Jayakody, S. K. Sharma, S. Chatzinotas, and J. Li, "Simultaneous wireless information and power transfer (SWIPT): Recent advances and future challenges," IEEE Commun. Surveys Tuts, vol. 20, no. 1, pp. 264-302, Firstquarter 2018.

[13] C. Zhu, V. C. M. Leung, L. Shu, and E. C. H. Ngai, "Green internet of things for smart world," IEEE Access, vol. 3, pp. 2151-2162, Nov. 2015.

[14] "Part 11: Wireless LAN Medium Access control (MAC) and Physical Layer (PHY) Specifications, IEEE standard 802.11," 2012.

[15] P. Liu, S. Gazor, I.-M. Kim, and D. Kim, "Noncoherent relaying in energy harvesting communication systems," IEEE Trans. Wireless Commun., vol. 14, no. 12, pp. 6940-6954, July 2015.

[16] - "Energy harvesting noncoherent cooperative communications," IEEE Trans. Wireless Commun., vol. 14, no. 12, pp. 6722-6737, July 2015.

[17] W. Xu, Z. Yang, Z. Ding, L. Wang, and P. Fan, "Wireless information and power transfer in two-way relaying network with non-coherent differential modulation," EURASIP J. on Wireless Commun. Netw., no. 131, May 2015.

[18] Y. Lou, Y. Zheng, J. Cheng, and H. Zhao, "Performance of SWIPTbased differential AF relaying over Nakagami- $m$ fading channels with direct link," IEEE Wireless Commun. Lett., vol. 7, no. 1, pp. 106-109, Feb. 2018.

[19] Y. Lou, Q. Y. Yu, J. Cheng, and H. L. Zhao, "Exact BER analysis of selection combining for differential SWIPT relaying systems," IEEE Signal Process. Lett., vol. 24, no. 8, pp. 1198-1202, Aug. 2017.

[20] L. Mohjazi, S. Muhaidat, and M. Dianati, "Performance analysis of differential modulation in SWIPT cooperative networks," IEEE Signal Process. Lett., vol. 23, no. 5, pp. 620-624, Feb. 2016.

[21] J. N. Laneman, D. N. C. Tse, and G. W.Wornell, "Cooperative diversity in wireless networks: Effcient protocols and outage behavior," IEEE Trans. Inform. Theory, vol. 50, no. 12, pp. 3062-3080, Dec. 2004.

[22] R. U. Nabar, H. Bolcskei, and F. W. Kneubuhler, "Fading relay channels: Performance limits and spacetime signal design," EEE J. Sel. Areas Commun., vol. 22, pp. 1099-1109, Aug. 2004.

[23] Q. Zhao and H. Li, "Differential modulation for cooperative wireless systems," IEEE Trans. Signal Process., vol. 55, no. 5, pp. 2273-2283, May 2007.

[24] J. G. Proakis, Digital Communications. New York: McGraw-Hill, 4th edition, 2000.

[25] POWERCAST. [Online]. Available: http://www.powercastco.com/

[26] A. P. Prudnikov, Y. A. Brychkov, and O. I. Marichev, Integrals and Series. Gordon and Breach Science Publishers, 1986, vol. 3.

[27] G. A. Baker and P. Graves-Morris, Padé Approximants. Cambridge, UK.: Cambridge Univ. Press, 1996.

[28] H. Amindavar and J. A. Ritcey, "Padé approximations of probability density functions," IEEE Trans. Aerosp. Elect. Syst., vol. 30, no. 2, pp. 416-424, Apr. 1994.

[29] M. K. Simon and M.-S. Alouini, Digital Communications over Fading Channels. A Unified Approach to Performance Analysis. New York, NY: John Wiley and Sons, Inc., 2000.

[30] Y.-C. Ko, M. S. Alouini, and M. K. Simon, "Outage probability of diversity systems over generalized fading channels," IEEE Trans. Commun., vol. 48, no. 11, pp. 1783-1787, Nov. 2000.

[31] M. K. Simon and M. S. Alouini, "A unified approach to the probability of error for noncoherent and differentially coherent modulations over generalized fading channels," IEEE Trans. Commun., vol. 46, no. 12, pp. 1625-1638, Dec. 1998.

[32] I. S. Ansari, F. Yilmaz, and M. S. Alouini, "Performance analysis of freespace optical links over malaga $(\mathcal{M})$ turbulence channels with pointing errors," IEEE Trans. Wireless Commun., vol. 15, no. 1, pp. 91-102, Aug. 2016.

[33] I. M. Ryzhik and I. S. Gradshteyn, Table of Integrals, Series, and Products. Academic Press, 7th edition, 2007. 